\title{
Mathematical Modelling of Bacterial Meningitis Transmission Dynamics with Control Measures
}

\author{
Joshua Kiddy K. Asamoah $\mathbb{D}^{1,2}$ Farai Nyabadza $\mathbb{D}^{1},{ }^{3}$ Baba Seidu $\mathbb{D}^{4},{ }^{4}$ \\ Mehar Chand, ${ }^{5}$ and Hemen Dutta ${ }^{6}$ \\ ${ }^{1}$ Department of Mathematics, Kwame Nkrumah University of Science and Technology, Kumasi, Ghana \\ ${ }^{2}$ African Institute for Mathematical Sciences, Biriwa, Ghana \\ ${ }^{3}$ Division of Mathematics, Stellenbosch University, Western Cape, South Africa \\ ${ }^{4}$ Department of Mathematics, University for Development Studies, Navrongo, Ghana \\ ${ }^{5}$ Department of Applied Sciences, Guru Kashi University, Bathinda, India \\ ${ }^{6}$ Department of Mathematics, Gauhati University, Guwahati 781014, India
}

Correspondence should be addressed to Joshua Kiddy K. Asamoah; jasamoah@aims.edu.gh

Received 23 September 2017; Accepted 4 January 2018; Published 27 March 2018

Academic Editor: Konstantin Blyuss

Copyright ( 2018 Joshua Kiddy K. Asamoah et al. This is an open access article distributed under the Creative Commons Attribution License, which permits unrestricted use, distribution, and reproduction in any medium, provided the original work is properly cited.

\begin{abstract}
Vaccination and treatment are the most effective ways of controlling the transmission of most infectious diseases. While vaccination helps susceptible individuals to build either a long-term immunity or short-term immunity, treatment reduces the number of disease-induced deaths and the number of infectious individuals in a community/nation. In this paper, a nonlinear deterministic model with time-dependent controls has been proposed to describe the dynamics of bacterial meningitis in a population. The model is shown to exhibit a unique globally asymptotically stable disease-free equilibrium $\mathscr{E}_{0}$, when the effective reproduction number $\mathscr{R}_{\mathrm{VT}} \leq 1$, and a globally asymptotically stable endemic equilibrium $\mathscr{E}_{1}$, when $\mathscr{R}_{\mathrm{VT}}>1$; and it exhibits a transcritical bifurcation at $\mathscr{R}_{\mathrm{VT}}=1$. Carriers have been shown (by Tornado plot) to have a higher chance of spreading the infection than those with clinical symptoms who will sometimes be bound to bed during the acute phase of the infection. In order to find the best strategy for minimizing the number of carriers and ill individuals and the cost of control implementation, an optimal control problem is set up by defining a Lagrangian function $L$ to be minimized subject to the proposed model. Numerical simulation of the optimal problem demonstrates that the best strategy to control bacterial meningitis is to combine vaccination with other interventions (such as treatment and public health education). Additionally, this research suggests that stakeholders should press hard for the production of existing/new vaccines and antibiotics and their disbursement to areas that are most affected by bacterial meningitis, especially Sub-Saharan Africa; furthermore, individuals who live in communities where the environment is relatively warm (hot/moisture) are advised to go for vaccination against bacterial meningitis.
\end{abstract}

\section{Introduction}

Meningitis is an inflammation of the meninges which are membranes that surround the spinal cord and the brain [1]. It is often caused by viruses, bacteria, and protozoa. Bacterial meningitis is common in children and young adults. This disease mostly spreads in communities/societies that live in close quarters (e.g., police staff, police cells, college students, military staff, and prisons) [2]. Bacterial meningitis is generally caused by germs such as Listeria monocytogenes, Streptococcus pneumoniae, Group B Streptococcus, Neisseria meningitidis, and Haemophilus influenzae, which spreads from one person to another [3]. This infection varies by age groups: Group B Streptococcus, Streptococcus pneumoniae, Listeria monocytogenes, and Escherichia coli are mostly found in newborn babies; Streptococcus pneumoniae, Neisseria meningitidis, Haemophilus influenzae type b (Hib), and Group B Streptococcus are common in babies and children; Neisseria meningitidis and Streptococcus pneumoniae are predominant in teens and young adults; and Streptococcus pneumoniae, Neisseria meningitidis, Haemophilus influenzae type b (Hib), Group B Streptococcus, and Listeria monocytogenes 
are commonly found in older adults [3]. Bacterial meningitis is characterized by intense headache and fever, vomiting, sensitivity to light, and stiff neck, which result in convulsion, delirium, and death.

It is estimated that meningococcal meningitis causes over 10,000 deaths annually in Sub-Saharan Africa [4]. About 4,100 cases of bacterial meningitis occurred between 2003 and 2007 in the United States [3, 5]. Between 5\% to $40 \%$ of children and $20 \%$ to $50 \%$ of adults with this condition die [6]. Infections from bacterial meningitis can cause permanent disabilities such as brain damage, hearing loss, and learning disabilities [3]. The illness of bacterial meningitis becomes worse when symptoms are not detected early enough; even with proper treatment, the individual could die [2].

Prevention of bacterial meningitis can be achieved through vaccination and/or preventing contact with infectious individuals. Vaccination is the most effective way of protecting children against certain types of bacterial meningitis [3]. Vaccines that can prevent meningitis include Haemophilus influenza type B (Hib), pneumococcal conjugate, and meningococcal vaccine $[6,7]$. The conjugate meningitis A vaccine, MenAfrivac, is recommended to protect people in Sub-Saharan Africa against the most common type, serotype A [8]. In the United States, the primary means of preventing meningococcal meningitis is antimicrobial chemoprophylaxis [9]. Empirical therapy includes ceftriaxone or cefotaxime and vancomycin for Streptococcus pneumoniae [2]. There is a vaccine against meningococcal disease which is $85 \%-100 \%$ effective in preventing four kinds of bacteria (serogroups A, C, Y, and W-135) that cause about $70 \%$ of the disease in the United States [2].

Trotter and Ramsay [10] outlined some recommendations on the use of conjugate vaccines in Europe based on the experience with meningococcal $\mathrm{C}$ conjugate (MCC) vaccines. In areas with limited health infrastructure and resources, there are a number of antibiotics including penicillin, ampicillin, and chloramphenicol that can be used to treat the infection meningitis.

Mathematical models have been shown to help increase the understanding of the spread and control of infectious diseases. Martínez et al. [2] studied the spread of meningococcal meningitis with the use of a discrete mathematical model, based on cellular automata where the population was divided into five classes: susceptible, asymptomatic infected, infected with symptoms, carriers, recovered, and died classes. Broutin et al. [11] studied the dynamics of meningococcal meningitis in nine African countries by adopting some mathematical tools to time series analysis and wavelet method, the results of their studies suggest that "international cooperation in Public Health and cross disciplines studies are highly recommended to help in controlling this infectious disease." Miller and Shahab [12] studied the cost effectiveness of immunisation strategies for the control of epidemic meningococcal meningitis. The research work in [13] gives a detailed description of the use of antibiotics for the prevention and treatment of meningitis infection. Irving et al. [14] used deterministic compartmental models to investigate how well simple model structures with seasonal forcing were able to qualitatively capture the patterns of meningitis infection. They demonstrated that the complex and irregular timing of epidemics could be caused by the interaction of temporary immunity conferred by carriage of the bacteria together with seasonal changes in the transmissibility of infection. Actually, there have been a significant number of studies of various types of Meningitis in Africa and Europe without the use of optimal control analysis (see [15-28]).

It is obvious that mathematical modelling has become crucial in investigating the epidemiological behaviour of meningitis. Furthermore, mathematical modelling helps to identify the risk factors for diseases, so as to find out why everyone does not have the same infection uniformly [29].

The application of optimal control in disease modelling gives valuable information on how to apply control measures. Through vaccination, treatment, public education, and so forth, many infectious diseases have been controlled [29]. Since the introduction of optimal control theory in disease modelling, there have been a considerable number of studies of infectious diseases using optimal control analysis (see [3041]). With the significant influence of optimal control theory in disease modelling, this paper presents an optimal control model for bacterial meningitis in the presence of vaccination and treatment due to public health education. The model is qualitatively analyzed and numerically simulated in order to help give policy direction on how to control the spread of the disease.

The rest of the paper is organized as follows. Section 2 presents the model formulation and analysis. Section 3 presents the analysis of the optimal control problem, leading to the existence and characterization of the control measures. Section 4 contains the numerical simulations and discussion. Section 5 presents the conclusion of the study.

\section{Model Formulation and Analysis}

Adopting the epidemiological studies of a meningitis model as presented in [4], we consider four mutually exclusive compartments to indicate individuals with unique natures (i.e., susceptibles, $S(t)$, carriers, $C(t)$, ill individuals, $I(t)$, and recovered individuals, $R(t))$ in relation to the disease. It is assumed that the susceptible compartment, $S(t)$, is populated through recruitment at the rate, $\pi$ (thus migration and/or birth rate), and $\beta$ is the rate of effective contact of carriers and/or infected (ill) individuals in the susceptible population. The carrier compartment consists of individuals that have the infection and do not show any clinical symptoms but contribute to the spread of the disease. When a susceptible individual is exposed to this infection, that individual can harbour the bacterium for weeks or even months [42]; but, in a normal circumstance, an individual develops symptoms of the infection within 3 to 7 days after exposure [3]. Carriers are assumed to develop clinical symptoms (i.e., move to ill individuals compartment, $I(t))$ at rate $\alpha$. Ill individuals who are seriously infected are assumed to have no natural recovery except when given treatment on time. From an epidemiological perspective, individuals in the removed/recovered compartment, $R(t)$, do not attain permanent immunity. After vaccination, immunity develops within 7-10 days and remains effective for approximately 3-5 years [2]. Therefore, 
it is assumed that the immunity acquired from developing the diseases or carrying the bacteria or through vaccination is of the same intensity (they all lead to the recovered compartment from which people return to the susceptible compartment at a given unique rate $\theta$ ). Research indicates that carriers may recover naturally from the infection without treatment, and we denote such natural recovery rate as $\omega$. Infected individuals are assumed to die from disease at rate $\delta$. Since natural death is inevitable, $\mu$ is assumed to be the natural death rate of individuals in all the compartments. Vaccination and treatment due to public health education have been shown to be strategies of control of diseases. Therefore we introduced this two control measures in the model as $u_{1}(t)$ and $u_{2}(t)$, respectively, here $u_{1}$; thus vaccination is comprised of both reactive vaccination and preventative vaccination. The effectiveness of both control measures in minimizing the disease is denoted by $\sigma$ and $\gamma$, respectively. If $\sigma=\gamma=0$, it signifies that vaccination and treatment have no effect on the model; if $\sigma=\gamma=1$, it also signifies that vaccination and treatment are perfectly effective (i.e., $100 \%$ effectiveness) (see [43]). If $0<\sigma$ and $\gamma<1$, it signifies that both vaccine and treatment are imperfect [44]. In view of this, it is assumed that administering treatment to the ill individuals leads to recovery at rate $\gamma u_{2}$. It is also assumed that the vaccinated individuals develop partial immunity at rate $\sigma u_{1}$. From an epidemiological perspective treatment is not given to carriers in real life (since we do not know who carries the bacteria or not), but it is assumed that carriers of meningitis are just like people with the HIV virus who do not know their status unless they go for medical test; hence this paper seeks to encourage individuals to go for regular test of this bacterial disease; therefore it is assumed that a certain portion of carriers could be treated before any symptoms of the infection show up, which results in $\gamma u_{2} C$ as shown in Figure 1. The vaccine is assumed to be imperfect and thus has a failure rate of $\left(1-u_{1} \sigma\right)$. Therefore the force of new infections is given by

$$
\lambda=\frac{\beta\left(1-u_{1} \sigma\right)\left(\eta C+\eta_{1} I\right)}{N} .
$$

Equation (1) is often referred to as standard incidence rate of new infections, which is normalized by the total population $N=S+C+I+R$. Table 1 gives a full description of parameters used in the model.

The set of differential equations and flow diagram corresponding to the bacterial meningitis dynamics and disease pathway with control terms is given in system (2) and Figure 1.

$$
\begin{aligned}
\frac{d S}{d t}= & \pi-\frac{\left(1-u_{1} \sigma\right) \beta S\left(\eta C+\eta_{1} I\right)}{N}-\left(\mu+u_{1} \sigma\right) S \\
& +\theta R, \\
\frac{d C}{d t}= & \frac{\left(1-u_{1} \sigma\right) \beta S\left(\eta C+\eta_{1} I\right)}{N} \\
& -\left(\alpha+\omega+\mu+u_{2} \gamma\right) C, \\
\frac{d I}{d t}= & \alpha C-\left(\delta+\mu+u_{2} \gamma\right) I,
\end{aligned}
$$

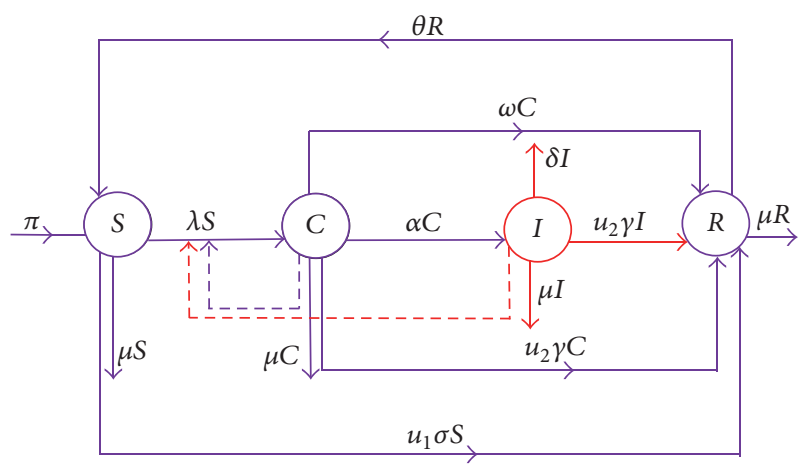

FIgURE 1: The flowchart diagram describing bacterial meningitis transmission dynamics within the population. The four circles represent the four compartments of individuals, the movement between the compartments is indicated by the continuous arrows, $u_{1}$ is a control measure (vaccination), and $u_{2}$ is the second control measure (treatment), with the consideration that both control measures lies in $0<u_{1}$ and $u_{2} \leq 1$.

$$
\begin{aligned}
& \frac{d R}{d t}=u_{2} \gamma I+\left(\omega+u_{2} \gamma\right) C+u_{1} \sigma S-(\theta+\mu) R \\
& \quad S(0)>0, C(0) \geq 0, I(0) \geq 0, R(0) \geq 0 .
\end{aligned}
$$

Adding the equations in model (2) gives the rate of change of total population as

$$
\frac{d N}{d t}=\pi-\mu N-\delta \mathrm{I} \leq \pi-\mu N
$$

Let

$$
\Omega=\left\{(S, C, I, R) \in \mathbb{R}_{+}^{4}: N \leq \frac{\pi}{\mu}\right\} .
$$

System (2) is well-posed with all solutions in $\Omega$ remaining in $\Omega$ if initial conditions are positive. It can easily be shown that if the initial conditions start outside $\Omega$ the solutions tend to $\Omega$.

2.1. Equilibrium Points. To obtain the equilibrium points of system (2), we assume that the control measures are timeindependent (see [45] for similar analysis).

2.1.1. Disease-Free Equilibrium $\mathscr{E}_{0}$. To obtain the disease-free equilibrium, $C(t), I(t)$, and the right-hand-side of system (2) are set to zero. If susceptible individuals are assumed to receive vaccination against the disease at a constant rate, then the disease-free equilibrium will be given by

$$
\begin{aligned}
\mathscr{E}_{0} & \left(S_{0}, C_{0}, I_{0}, R_{0}\right) \\
= & \left(\frac{\pi(\theta+\mu)}{\mu\left(u_{1} \sigma+\theta+\mu\right)}, 0,0, \frac{\pi u_{1} \sigma}{\mu\left(u_{1} \sigma+\theta+\mu\right)}\right) .
\end{aligned}
$$

2.1.2. Effective Reproduction Number $\mathscr{R}_{V T}$. Using the next generation matrix method [46], the effective reproduction 


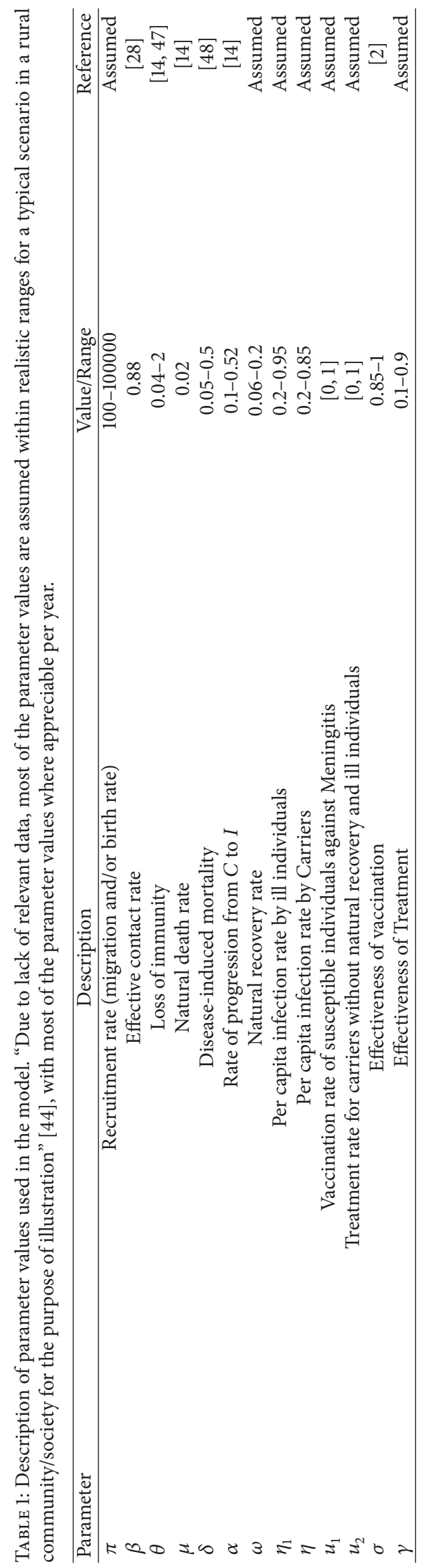




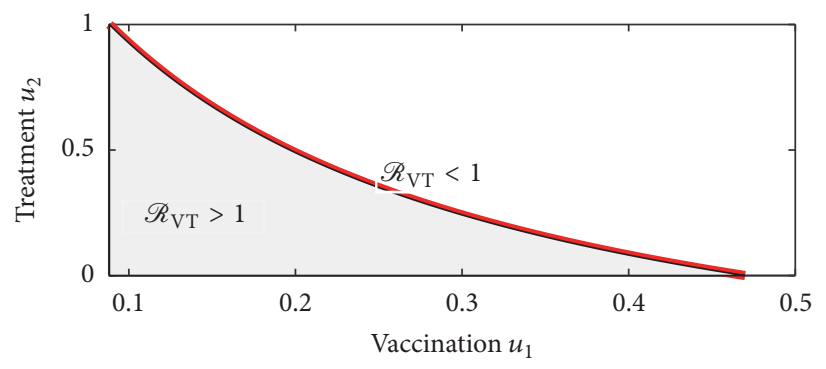

FIGURE 2: Region where $\mathscr{R}_{\mathrm{VT}}<1$ and $\mathscr{R}_{\mathrm{VT}}>1$ in the $u_{1}-u_{2}$ parameter space, with the parameter values $\beta=0.88, \eta=0.2, \omega=0.06, \delta=$ $0.03, \theta=0.0839, \alpha=0.05, \gamma=0.2$ and $\sigma=0.7$.

number of the bacterial meningitis model with vaccination and treatment is obtained as

$$
\mathscr{R}_{\mathrm{VT}}=\mathscr{R}_{C_{\mathrm{VT}}}+\mathscr{R}_{I_{\mathrm{VT}}},
$$

where

$$
\begin{aligned}
& \mathscr{R}_{C_{\mathrm{VT}}}=\frac{\beta \eta\left(1-u_{1} \sigma\right)(\theta+\mu)}{\left(\alpha+\omega+\mu+u_{2} \gamma\right)\left(\theta+\mu+u_{1} \sigma\right)}, \\
& \mathscr{R}_{I_{\mathrm{VT}}} \\
& \quad=\frac{\beta \alpha \eta_{1}\left(1-u_{1} \sigma\right)(\theta+\mu)}{\left(\alpha+\omega+\mu+u_{2} \gamma\right)\left(\delta+\mu+u_{2} \gamma\right)\left(\theta+\mu+u_{1} \sigma\right)} .
\end{aligned}
$$

2.1.3. Endemic Equilibrium $\mathscr{E}_{1}$. System (2) can be shown to have a unique endemic equilibrium of the form $\left(S^{*}, C^{*}, I^{*}, R^{*}\right)$, where
To determine how the two control measures impact on the reproduction number, we make a plot of $\mathscr{R}_{\mathrm{VT}}$, on the $u_{1}-u_{2}$ plane for arbitrary constant values of model parameters in Figure 2. From the figure, it is shown that $u_{2}$ decreases with increasing $u_{1}$. So an increase in vaccination levels decreases the need for treatment. Figure 2 also shows the region in which the vaccination and treatment values should lie for the disease control.

$$
\begin{aligned}
& S^{*}=\frac{\alpha \pi(\mu+\theta)+\left[\left(\omega+u_{2} \gamma\right)\left(\delta+\mu+u_{2} \gamma\right) \theta+\theta \alpha u_{2} \gamma-\left(\alpha+\omega+\mu+u_{2} \gamma\right)\left(\delta+\mu+u_{2} \gamma\right)(\theta+\mu)\right] \pi \alpha\left(\mathscr{R}_{\mathrm{VT}}-1\right)}{\alpha \mu\left(\theta+\mu+u_{1} \sigma\right)\left[\mathbf{A}\left(\mathscr{R}_{\mathrm{VT}}-1\right)+\mathbf{B}\right]}, \\
& C^{*}=\frac{\left(\delta+\mu+u_{2} \gamma\right)\left[\pi \alpha\left(\mathscr{R}_{\mathrm{VT}}-1\right)\right]}{\alpha\left[\mathbf{A}\left(\mathscr{R}_{\mathrm{VT}}-1\right)+\mathbf{B}\right]}, \\
& R^{*} \\
& =\frac{\alpha \pi u_{1} \sigma+\left[\left(\delta+\mu+u_{2} \gamma\right)\left(\omega+u_{2} \gamma\right)\left(\mu+u_{1} \sigma\right)+\alpha u_{2} \gamma\left(\mu+u_{1} \sigma\right)-\left(\alpha+\omega+\mu+u_{2} \gamma\right)\left(\delta+\mu+u_{2} \gamma\right) u_{1} \sigma\right]\left[\pi \alpha\left(\mathscr{R}_{\mathrm{VT}}-1\right)\right]}{\alpha \mu\left(\theta+\mu+u_{1} \sigma\right)\left[\mathbf{A}\left(\mathscr{R}_{\mathrm{VT}}-1\right)+\mathbf{B}\right]}, \\
& I^{*}=\frac{\pi \alpha\left(\mathscr{R}_{\mathrm{VT}}-1\right)}{\mathbf{A}\left(\mathscr{R}_{\mathrm{VT}}-1\right)+\mathbf{B}},
\end{aligned}
$$

with $\mathbf{A}=\left(\delta+\mu+u_{2} \gamma\right)\left(\alpha+\omega+\mu+u_{2} \gamma\right)$ and $\mathbf{B}=(\delta+\mu+$ $\left.\left.u_{2} \gamma\right)\left[\omega+u_{2} \gamma-u_{1} \sigma\right)+\alpha\left(u_{2} \gamma+\mu\right)\right]$.

Remark 1. (i) If $\mathscr{R}_{\mathrm{VT}}<1$, then system (2) will have only one equilibrium: the disease-free equilibrium.

(ii) If $\mathscr{R}_{\mathrm{VT}}>1$, then system (2) will have two equilibria: the disease-free equilibrium, $\mathscr{E}_{0}$, and the endemic equilibrium, $\mathscr{E}_{1}$.

(iii) The case $\mathscr{R}_{\mathrm{VT}}=1$ is a critical threshold point where the disease-free equilibrium $\mathscr{E}_{0}$ loses its local asymptotic stability. Thus $\mathscr{R}_{\mathrm{VT}}=1$ gives the idea of transcritical bifurcation where the stability of system (2) moves between $\mathscr{E}_{0}$ and $\mathscr{E}_{1}$ [37]. 


$$
J\left(\mathscr{E}_{0}\right)=\left[\begin{array}{cccc}
-\left(\mu+u_{1} \sigma\right) & -\frac{\left(1-u_{1} \sigma\right) \beta \eta(\theta+\mu)}{\left(u_{1} \sigma+\theta+\mu\right)} & -\frac{\left(1-u_{1} \sigma\right) \beta \eta_{1}(\theta+\mu)}{\left(u_{1} \sigma+\theta+\mu\right)} & \theta \\
0 & \frac{\left(1-u_{1} \sigma\right) \beta \eta(\theta+\mu)}{\left(u_{1} \sigma+\theta+\mu\right)}-\left(\alpha+\omega+\mu+u_{2} \gamma\right) & \frac{\left(1-u_{1} \sigma\right) \beta \eta_{1}(\theta+\mu)}{\left(u_{1} \sigma+\theta+\mu\right)} & 0 \\
0 & \alpha & -\left(\delta+\mu+u_{2} \gamma\right) & 0 \\
u_{1} \sigma & \left(\omega+u_{2} \gamma\right) & u_{2} \gamma & -(\theta+\mu)
\end{array}\right] .
$$

The characteristic polynomial of the Jacobian matrix $J\left(\mathscr{E}_{0}\right)$ is given by

$$
P(\lambda)=\lambda^{4}+\mathbf{D T} \lambda^{3}+\mathbf{D T}_{1} \lambda^{2}+\mathbf{D T}_{2} \lambda+\mathbf{D T}_{3},
$$

where

$$
\begin{aligned}
& \mathbf{T}=A+B+C-\mathscr{R}_{C_{\mathrm{VT}}}, \\
& \mathbf{T}_{1} \\
& =\left(A+B-\mathscr{R}_{C_{\mathrm{VT}}}\right)\left(2 \mu+u_{1} \sigma+\theta\right) \\
& +\left(\mu+u_{1} \sigma\right)(\theta+\mu)+\mu \theta\left(1-\mathscr{R}_{\mathrm{VT}}\right), \\
& =(\theta+\mu)\left[\left(1-\mathscr{R}_{\mathrm{VT}}\right)+\left(A-\mathscr{R}_{\mathrm{C}_{\mathrm{VT}}}\right)\right]-\mathscr{R}_{\mathrm{C}_{\mathrm{VT}}} \\
& +\left(\mu+u_{1} \sigma\right)\left[\left(1-\mathscr{R}_{I_{\mathrm{VT}}}\right)+\left(\delta+\mu+u_{2} \gamma\right)(\theta+\mu)\right] \\
& +\left(A+B-\mathscr{R}_{C_{\mathrm{VT}}}\right) \mu \theta \text {, } \\
& \mathbf{T}_{3}=\theta \mu\left(\mathscr{R}_{I_{\mathrm{VT}}}-1\right)+\left(\mu+u_{1} \sigma\right)(\theta+\mu)\left(\mathscr{R}_{I_{\mathrm{VT}}}-1\right) \text {, } \\
& A=\frac{1}{\left(\delta+\mu+u_{2} \gamma\right)}, \\
& B=\frac{1}{\left(\alpha+\omega+\mu+u_{2} \gamma\right)}, \\
& C=\frac{\left(\mu+u_{1} \sigma\right)+(\theta+\mu)}{\left(\alpha+\omega+\mu+u_{2} \gamma\right)\left(\delta+\mu+u_{2} \gamma\right)}, \\
& \mathbf{D}=\left(\alpha+\omega+\mu+u_{2} \gamma\right)\left(\delta+\mu+u_{2} \gamma\right) \text {. }
\end{aligned}
$$

The Routh-Hurwitz conditions [46] that guarantee that the eigenvalues of the characteristic polynomial in (10) have negative real parts are given by

$$
\begin{aligned}
\mathbf{D T} & >0, \\
\mathbf{D T}_{1} & >0, \\
\mathbf{D T}_{2} & >0, \\
\mathbf{D T}_{3} & >0 \\
\mathbf{D}^{3} \mathbf{T T}_{1} \mathbf{T}_{2} & >\mathbf{D}^{2} \mathbf{T}_{2}^{2}+\mathbf{D}^{3} \mathbf{T}^{2} \mathbf{T}_{3} .
\end{aligned}
$$

These conditions are easily seen to be satisfied when $\mathscr{R}_{\mathrm{VT}}<1$. Thus, the disease-free equilibrium of system (2) is locally asymptotically stable when $\mathscr{R}_{\mathrm{VT}}<1$ and unstable when $\mathscr{R}_{\mathrm{VT}}>1$. This completes the proof.

\subsubsection{Global Stability of $\mathscr{E}_{0}$}

Theorem 3. The disease-free equilibrium $\mathscr{E}_{0}$ of system (2) is globally asymptotically stable if $\mathscr{R}_{V T} \leq 1$ and unstable if $\mathscr{R}_{V T}>1$.

Proof. Let $\mathscr{V}(S, C, I, R)$, with positive constants, $\mathscr{K}_{1}$ and $\mathscr{K}_{2}$, be a Lyapunov function defined as

$$
\begin{aligned}
\mathscr{V}(\mathrm{S}, C, I, R)= & \left(S-S_{0}-S_{0} \ln \frac{S}{S_{0}}\right)+\mathscr{K}_{1} C+\mathscr{K}_{2} I \\
& +\left(R-R_{0}-R_{0} \ln \frac{R}{R_{0}}\right) .
\end{aligned}
$$

Taking the time derivative of the Lyapunov function we obtain

$$
\begin{aligned}
\frac{d \mathscr{V}}{d t}= & \left(1-\frac{S_{0}}{S}\right) \frac{d S}{d t}+\mathscr{K}_{1} \frac{d C}{d t}+\mathscr{K}_{2} \frac{d I}{d t} \\
& +\left(1-\frac{R_{0}}{R}\right) \frac{d R}{d t}, \quad \text { where } \mathscr{K}_{1} \geq 0, \mathscr{K}_{2} \geq 0 .
\end{aligned}
$$

Substituting $d S / d t, d C / d t, d I / d t$, and $d R / d t$ in (2) into (14) gives

$$
\begin{aligned}
& \frac{d \mathscr{V}}{d t}=\left(1-\frac{S}{S_{0}}\right)\left[\pi-\frac{\left(1-u_{1} \sigma\right) \beta S\left(\eta C+\eta_{1} I\right)}{N}\right. \\
& \left.-\left(\mu+\sigma u_{1}\right) S+\theta R\right] \\
& +\mathscr{K}_{1}\left[\frac{\left(1-u_{1} \sigma\right) \beta S\left(\eta C+\eta_{1} I\right)}{N}\right. \\
& \left.-\left(\alpha+\omega+\mu+u_{2} \gamma\right) C\right]+\mathscr{K}_{2}[\alpha C \\
& \left.-\left(\delta+\mu+u_{2} \gamma\right) I\right]+\left(1-\frac{R}{R_{0}}\right)\left[u_{2} \gamma I\right.
\end{aligned}
$$




$$
\begin{aligned}
& \left.+\left(\omega+u_{2} \gamma\right) C+\sigma u_{1} S-(\theta+\mu) R\right] \\
& \leq \mathscr{K}_{1}\left[\left(\frac{\left(1-u_{1} \sigma\right) \beta \eta(\theta+\mu)}{\left(u_{1} \sigma+\theta+\mu\right)}\right) C\right. \\
& +\left(\frac{\left(1-u_{1} \sigma\right) \beta \eta_{1}(\theta+\mu)}{\left(u_{1} \sigma+\theta+\mu\right)}\right) I \\
& \left.-\left(\alpha+\omega+\mu+u_{2} \gamma\right) C\right]+\mathscr{K}_{2}[\alpha C \\
& \left.-\left(\delta+\mu+u_{2} \gamma\right) I\right] .
\end{aligned}
$$

Since

$$
\begin{aligned}
& S \leq S_{0}=\frac{\pi(\theta+\mu)}{\mu\left(u_{1} \sigma+\theta+\mu\right)}, \\
& R \leq R_{0}=\frac{\pi u_{1} \sigma}{\mu\left(u_{1} \sigma+\theta+\mu\right)}, \\
& N \leq \frac{\pi}{\mu}
\end{aligned}
$$

on $\Omega$,

this implies that

$$
\begin{aligned}
\frac{d \mathscr{V}}{d t} & \leq\left[\left(\frac{\left(1-u_{1} \sigma\right) \beta \eta(\theta+\mu)}{\left(u_{1} \sigma+\theta+\mu\right)}\right) \mathscr{K}_{1}+\alpha \mathscr{K}_{1}\right. \\
& \left.-\left(\alpha+\omega+\mu+u_{2} \gamma\right) \mathscr{K}_{2}\right] C \\
& +\left[\left(\frac{\left(1-u_{1} \sigma\right) \beta \eta_{1}(\theta+\mu)}{\left(u_{1} \sigma+\theta+\mu\right)}\right) \mathscr{K}_{1}\right. \\
& \left.-\left(\delta+\mu+u_{2} \gamma\right) \mathscr{K}_{2}\right] I .
\end{aligned}
$$

Equating the coefficient of $I$ in (17) to zero gives

$$
\begin{aligned}
(1 & \left.-u_{1} \sigma\right) \beta(\theta+\mu) \mathscr{K}_{1} \\
& =\left(\delta+\mu+u_{2} \gamma\right)\left(u_{1} \sigma+\theta+\mu\right) \mathscr{K}_{2} .
\end{aligned}
$$

Choosing $\mathscr{K}_{1}=\left(\delta+\mu+u_{2} \gamma\right)\left(u_{1} \sigma+\theta+\mu\right)$ and $\mathscr{K}_{2}=(1-$ $\left.u_{1} \sigma\right) \beta(\theta+\mu)$ and plugging $\mathscr{K}_{1}$ and $\mathscr{K}_{2}$ into (17), we have

$$
\begin{gathered}
\frac{d \mathscr{V}}{d t} \leq\left(\alpha+\omega+\mu+u_{2} \gamma\right)\left(\delta+\mu+u_{2} \gamma\right)\left(u_{1} \sigma+\theta+\mu\right) \\
\cdot\left(\mathscr{R}_{\mathrm{VT}}-1\right) C \leq 0, \quad \text { if } \mathscr{R}_{\mathrm{VT}} \leq 1 .
\end{gathered}
$$

Additionally $d \mathscr{V} / d t=0$ if and only if $C=0$. Hence, the largest compact invariant set in $\{(S, C, I, R) \in \Omega$ : $d \mathscr{V} / d t \leq 0\}$ is the singleton set $\left\{\mathscr{E}_{0}\right\}$. Therefore, from LaSalle's invariance principle, we conclude that $\mathscr{E}_{0}$ is globally asymptotically stable in $\Omega$ if $\mathscr{R}_{\mathrm{VT}} \leq 1[37,49]$.

\subsubsection{Global Stability of $\mathscr{E}_{1}$}

Theorem 4. The endemic equilibrium $\mathscr{E}_{1}$ of system (2) is globally asymptotically stable whenever $\mathscr{R}_{V T}>1$.

Proof. Suppose $\mathscr{R}_{\mathrm{VT}}>1$, and then the existence of the endemic equilibrium point is assured. Using the common quadratic Lyapunov function

$$
V\left(x_{1}, x_{2}, \ldots, x_{n}\right)=\sum_{i=1}^{n} \frac{c_{i}}{2}\left(x_{i}-x_{i}^{*}\right)^{2},
$$

as illustrated in [50], we consider the following candidate Lyapunov function:

$$
\begin{aligned}
\mathscr{V} & (S, C, I, R) \\
& =\frac{1}{2}\left[\left(S-S^{*}\right)+\left(C-C^{*}\right)+\left(I-I^{*}\right)+\left(R-R^{*}\right)\right]^{2} .
\end{aligned}
$$

The time derivative of $\mathscr{V}(S, C, I, R)$ in (21) is given by

$$
\begin{aligned}
& \frac{d \mathscr{V}}{d t}(S, C, I, R)=\left[\left(S-S^{*}\right)+\left(C-C^{*}\right)+\left(I-I^{*}\right)\right. \\
& \left.\quad+\left(R-R^{*}\right)\right] \frac{d(S+C+I+R)}{d t} .
\end{aligned}
$$

Plugging the equations in system (2) into (22) yields

$$
\begin{aligned}
& \frac{d \mathscr{V}}{d t}=\left[\left(S-S^{*}\right)+\left(C-C^{*}\right)+\left(I-I^{*}\right)+\left(R-R^{*}\right)\right] \\
& \cdot[\pi-\mu(S+C+I+R)-\delta I] .
\end{aligned}
$$

Now setting

$$
\pi=\mu\left(S^{*}+C^{*}+I^{*}+R^{*}\right)+\delta I^{*},
$$

we have

$$
\begin{aligned}
& \frac{d \mathscr{V}}{d t}=\left[\left(S-S^{*}\right)+\left(C-C^{*}\right)+\left(I-I^{*}\right)+\left(R-R^{*}\right)\right] \\
& \cdot\left[\mu\left(S^{*}+C^{*}+I^{*}+R^{*}\right)+\delta I^{*}\right. \\
& -\mu(S+C+I+R)-\delta I]=\left[\left(S-S^{*}\right)+\left(C-C^{*}\right)\right. \\
& \left.\quad+\left(I-I^{*}\right)+\left(R-R^{*}\right)\right]\left[-\mu\left(S-S^{*}\right)-\mu\left(C-C^{*}\right)\right. \\
& \left.\quad-\mu\left(I-I^{*}\right)-\mu\left(R-R^{*}\right)-\delta\left(I-I^{*}\right)\right] .
\end{aligned}
$$


Further simplification gives

$$
\begin{aligned}
& \frac{d \mathscr{V}}{d t}=-\mu\left(S-S^{*}\right)^{2}-\mu\left(S-S^{*}\right) \\
& \cdot\left[\left(C-C^{*}\right)+\left(I-I^{*}\right)+\left(R-R^{*}\right)+\delta\left(I-I^{*}\right)\right] \\
& -\mu\left(C-C^{*}\right)^{2}-\mu\left(C-C^{*}\right) \\
& \cdot\left[\left(S-S^{*}\right)+\left(I-I^{*}\right)+\left(R-R^{*}\right)+\delta\left(I-I^{*}\right)\right] \\
& -(\mu+\delta)\left(I-I^{*}\right)^{2}-\mu\left(I-I^{*}\right) \\
& \cdot\left[\left(C-C^{*}\right)+\left(R-R^{*}\right)\right]-\mu\left(R-R^{*}\right)^{2} \\
& -\mu\left(R-R^{*}\right) \\
& \cdot\left[\left(S-S^{*}\right)+\left(C-C^{*}\right)+\left(I-I^{*}\right)+\delta\left(I-I^{*}\right)\right] .
\end{aligned}
$$

It has therefore been shown that $d \mathscr{V} / d t$ is negative, and additionally at $\mathscr{E}_{1}$ (i.e., if $S=S^{*}, C=C^{*}, I=I^{*}$, and $R=R^{*}$ ), $d \mathscr{V} / d t=0$. It follows from LaSalle's invariant principle [51] that all solutions of system (2) approach $\mathscr{E}_{1}$ as $t \rightarrow \infty$ if $\mathscr{R}_{\mathrm{VT}}>1$. Therefore, the endemic equilibrium $\mathscr{E}_{1}$ is globally asymptotically stable in $\Omega$ whenever $\mathscr{R}_{\mathrm{VT}}>1[37,49]$. This completes the proof.

2.3. Sensitivity Analysis. Sensitivity analysis is used to determine the response of a model to variations in its parameter values. In the present case, the focus is given to determining how changes in the model parameters impact the effective reproduction number. This is done through the normalized forward-sensitivity index. We also use the Latin hypercube sampling and the partial rank correlation coefficients (PRCC) to plot scatter diagrams and Tornado plots to determine the relative importance of the parameters in $\mathscr{R}_{\mathrm{VT}}$ for the disease transmission and prevalence (see also [52]).

Definition 5. The normalized forward-sensitivity index of $\mathscr{R}_{\mathrm{VT}}$ to any parameter, say $\rho$, as given in [46] can be defined as

$$
\Gamma_{\mathscr{R}_{\mathrm{VT}}}^{\rho}=\frac{\partial \mathscr{R}_{\mathrm{VT}}}{\partial \rho} \frac{\rho}{\mathscr{R}_{\mathrm{VT}}} .
$$

The sensitivity indexes of $\mathscr{R}_{\mathrm{VT}}$ with respect to its parameters are computed as follows:

$$
\begin{aligned}
\Gamma_{\mathscr{R}_{\mathrm{VT}}}^{\beta} & =\frac{\partial \mathscr{R}_{\mathrm{VT}}}{\partial \beta} \frac{\beta}{\mathscr{R}_{\mathrm{VT}}}=1, \\
\Gamma_{\mathscr{R}_{\mathrm{VT}}}^{\eta} & =\frac{\partial \mathscr{R}_{\mathrm{VT}}}{\partial \eta} \frac{\eta}{\mathscr{R}_{\mathrm{VT}}}=\frac{\left(\delta+\mu+u_{2} \gamma\right) \eta}{\eta\left(\delta+\mu+u_{2} \gamma\right)+\eta_{1} \alpha}, \\
\Gamma_{\mathscr{R}_{\mathrm{VT}}}^{\eta_{1}} & =\frac{\partial \mathscr{R}_{\mathrm{VT}}}{\partial \eta_{1}} \frac{\eta_{1}}{\mathscr{R}_{\mathrm{VT}}}=\frac{\alpha \eta_{1}}{\left(\alpha \eta_{1}+\left(\delta+\mu+u_{2} \gamma\right)\right)}, \\
\Gamma_{\mathscr{R}_{\mathrm{VT}}}^{\omega} & =\frac{\partial \mathscr{R}_{\mathrm{VT}}}{\partial \omega} \frac{\omega}{\mathscr{R}_{\mathrm{VT}}}=-\frac{\omega}{\left(\alpha+\omega+\mu+u_{2} \gamma\right)}, \\
\Gamma_{\mathscr{R}_{\mathrm{VT}}}^{\sigma} & =\frac{\partial \mathscr{R}_{\mathrm{VT}}}{\partial \sigma} \frac{\sigma}{\mathscr{R}_{\mathrm{VT}}}=-\frac{u_{1} \sigma}{\left(u_{1} \sigma+\theta+\mu\right)} .
\end{aligned}
$$

Similarly, we can compute the sensitivity indexes of $\mathscr{R}_{\mathrm{VT}}$ with respect to the remaining parameters in $\mathscr{R}_{\mathrm{VT}}$, in the same manner. Using the parameter values $\gamma=0.4, \sigma=1, \eta=0.35$, $\omega=0.2, \delta=0.1, \beta=0.88, \alpha=0.2, \theta=0.0839$, and $\mu=0.02$, with $u_{1}=0.5$ and $u_{2}=0.5$, the sensitivity indexes of $\mathscr{R}_{\mathrm{VT}}$ are shown in Table 2.

The corresponding Tornado plots based on a random sample of 1000 points for the twelve parameters in $\mathscr{R}_{\mathrm{VT}}$ are shown in Figure 3(a). The positive values in Table 2 show a promotion of the propagation of the disease. Therefore an increase in the values of $\beta, \eta, \eta_{1}, \theta$, and $\alpha$ will have an increase in the spread of the disease. For example, $\Gamma_{\mathscr{R}_{\mathrm{VT}}}^{\beta}=1$ indicates that increasing the effective contact rate by $10 \%$ increases the number of secondary infections by $10 \%$. The negative values in Table 2 indicate a reduction in the effective reproduction number $\mathscr{R}_{\mathrm{VT}}$ if the values of the corresponding parameters are increased. Thus, a reduction in the values of vaccination $u_{1}$, treatment $u_{2}$, and natural recovery $\omega$ will lead to an increase in the number of secondary infections in the population.

Figure 3(a) shows the Tornado plots for the twelve parameters in $\mathscr{R}_{\mathrm{VT}}$. It can be seen that, in controlling the spread of bacterial meningitis in a population, more susceptible individuals should be given vaccination. Figure 3(a) also suggests that carriers are likely to have more contacts with the susceptible population than the ill individuals who will typically be bound to their beds during the acute phase of the disease. Therefore, the probability of ill individuals transmitting the infections to susceptibles may be lower than that of carriers who are able to mix well with others within the population. Figures 3(b), 3(c), and 3(d) show the regression plots of effective contact rate $(\beta)$, vaccination rate $\left(u_{1}\right)$, and treatment rate $\left(u_{2}\right)$, respectively. Figure $3(\mathrm{~b})$ shows that transmission rate has a positive correlation in the spread of bacterial meningitis. Figure 3(c) shows that vaccination has a negative correlation in the spread of bacterial meningitis and hence vaccination increases the immunity of individuals against the meningitis infection, thereby reducing the spread of the infection. Figure 3(d) shows that individuals who receive treatment after being infected with bacterial meningitis have a higher chance of recovery and that reduces the spread of the infection and deaths due to bacterial meningitis.

\section{Optimal Control Problem}

Since the goal of this paper is to find the best ways to control the spread of meningitis, we define the following optimal control problem:

$$
\begin{aligned}
J= & \min _{u_{1} \sigma, u_{2}} \int_{0}^{T}\left[A_{1} C(t)+A_{2} I(t)+\frac{B_{1}}{2} u_{1}^{2}(t)\right. \\
& \left.+\frac{B_{2}}{2} u_{2}^{2}(t)\right] d t,
\end{aligned}
$$

subject to model (2). 
TABLE 2: Sensitivity indexes of $\mathscr{R}_{\mathrm{VT}}$ to the parameters in (6).

\begin{tabular}{lcc}
\hline Parameter & Description & Sensitivity index \\
\hline$\beta$ & Effective contact rate & 1 \\
$\eta$ & Per capita infection rate by Carriers & 0.6154 \\
$\eta_{1}$ & Per capita infection rate by ill individuals & 0.3846 \\
$\theta$ & Loss of immunity & 0.6686 \\
$\alpha$ & Rate of falling ill & 0.0620 \\
$\mu$ & Natural death rate & 0.1031 \\
$\delta$ & Disease-induced death rate & -0.1202 \\
$\omega$ & Natural recovery rate & -0.3226 \\
$u_{1}$ & Vaccination rate & -1.8280 \\
$\sigma$ & Effectiveness of vaccination & -0.8280 \\
$u_{2}$ & Treatment rate & -0.5630 \\
$\gamma$ & Effectiveness of treatment & -0.5230 \\
\hline
\end{tabular}

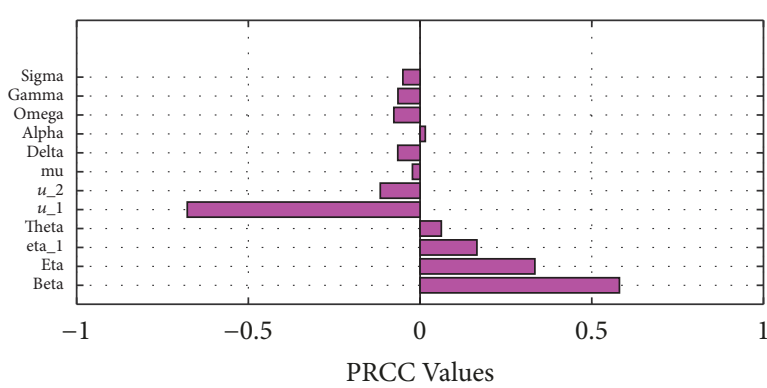

(a) The Tornado plots for the elven parameters in $\mathscr{R}_{\mathrm{VT}}$

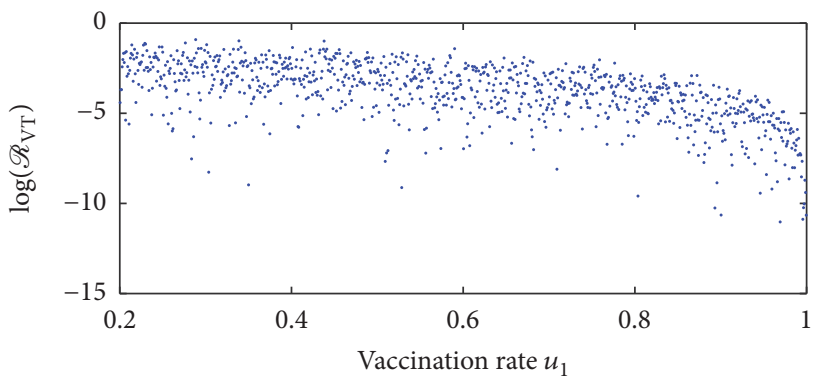

(c) The effect of vaccination on $\mathscr{R}_{\mathrm{VT}}$

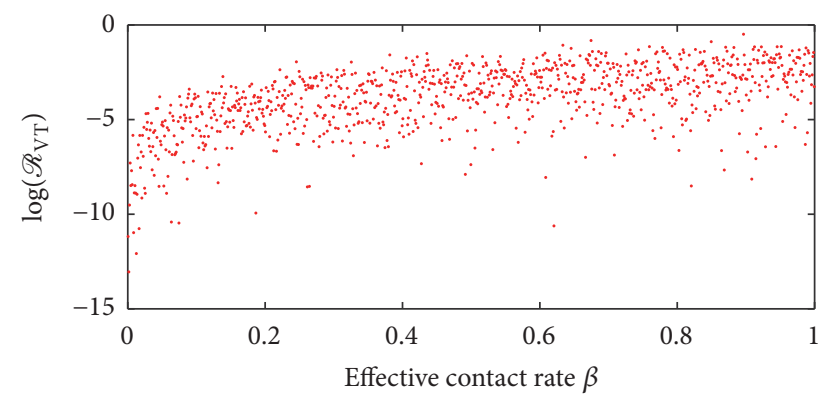

(b) The effect of effective contact rate on $\mathscr{R}_{\mathrm{VT}}$

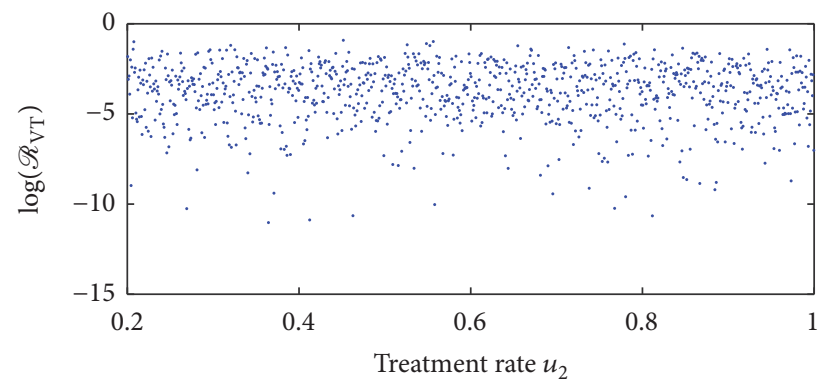

(d) The effect of treatment on $\mathscr{R}_{\mathrm{VT}}$

FIGURE 3: Sensitivity plots.

The admissible control set $U$ is Lebesgue measurable, which is defined by

$$
\begin{aligned}
U & =\left\{\left(u_{1}(t), u_{2}(t)\right) \mid 0 \leq u_{1} \leq u_{1 \max } \leq 1,0 \leq u_{2}\right. \\
& \left.\leq u_{2 \max } \leq 1, t \in[0, T]\right\} .
\end{aligned}
$$

Our objective is to find $\left(u_{1}^{*}, u_{2}^{*}\right) \in U$ which minimizes the associated cost of the vaccination and the associated cost of the treatment over the specified time interval, as well as minimizing the number of infections at a terminal time (see also [37]). The coefficients $A_{1}>0$ and $A_{2}>0$ are constants that are introduced to maintain a balance in the size of $C(t)$ and $I(t)$, respectively. $B_{1}>0$ and $B_{2}>0$ are the corresponding weights associated with the cost of vaccination $\left(u_{1}\right)$ and treatment $\left(u_{2}\right)$, respectively. The higher bounds (maximum) attainable for the control measures $u_{1} \sigma$ and $u_{2}$ are $u_{1 \max }$ and $u_{2 \max }$, respectively. We fix the control measures $u_{1}$ and $u_{2}$ to lie between 0 and 1 so that $u_{1 \max }=1$ and $u_{2 \max }=1$. Therefore the attainment of $u_{1 \max }$ and $u_{2 \max }$ depends on the number of resources available [37]. These resources may include the human effort, material resources, cost of producing vaccine and disbursement, infrastructural resources, the number of health facilities in the community, and the number of hospital beds at the health facilities. The cost of hospitalization, medical test, diagnosis, drug cost, and so forth (see [38-40]) can be associated with treatment. The 
cost of vaccination may include the cost of the vaccine, the cost of production, the cost of disbursement, the vaccine storage cost, and other related overheads [37]. The severity of the side effects and overdoses of the vaccination and treatment is taken care of by squaring the control measures, and $T$ is the final time during the optimal simulation.
3.1. Existence of the Optimal Control. Model (2) can be written as

$$
G_{t}=K(G)+F(G),
$$

where

$$
\begin{aligned}
& G=\left[\begin{array}{l}
S(t) \\
C(t) \\
I(t) \\
R(t)
\end{array}\right] \\
& K=\left[\begin{array}{cccc}
-\left(\mu+u_{1} \sigma\right) & 0 & 0 & \theta \\
0 & -\left(\alpha+\omega+\mu+u_{2} \gamma\right) & 0 & 0 \\
0 & \alpha & -\left(\delta+\mu+u_{1} \sigma\right) & 0 \\
u_{1} \sigma & \left(\omega+u_{2} \gamma\right) & u_{2} \gamma & -(\theta+\mu)
\end{array}\right] \text {, } \\
& F(G)=\left[\begin{array}{c}
\pi-\frac{\left(1-u_{1} \sigma\right) \beta S(t)\left(\eta C(t)+\eta_{1} I(t)\right)}{N(t)} \\
\frac{\left(1-u_{1} \sigma\right) \beta S(t)\left(\eta C(t)+\eta_{1} I(t)\right)}{N(t)} \\
0 \\
0
\end{array}\right] \text {, }
\end{aligned}
$$

and $G_{t}$ is the times derivative of $G(t)$. System (31) is nonlinear with a bounded coefficient.
Setting $G_{1}=\left(S_{1}(t), C_{1}(t), I_{1}(t), R_{1}(t)\right)$ and $G_{2}=\left(S_{2}(t)\right.$, $\left.C_{2}(t), I_{2}(t), R_{2}(t)\right)$ gives

$$
F\left(G_{1}\right)-F\left(G_{2}\right)=\left[\begin{array}{c}
-\left(1-u_{1} \sigma\right) \beta \eta\left(\frac{S_{1}(t) C_{1}(t)}{N_{1}(t)}-\frac{S_{2}(t) C_{2}(t)}{N_{2}(t)}\right)-\left(1-u_{1} \sigma\right) \beta\left(\frac{S_{1}(t) I_{1}(t)}{N_{1}(t)}-\frac{S_{2}(t) I_{2}(t)}{N_{2}(t)}\right) \\
\left(1-u_{1} \sigma\right) \beta \eta\left(\frac{S_{1}(t) C_{1}(t)}{N_{1}(t)}-\frac{S_{2}(t) C_{2}(t)}{N_{2}(t)}\right)-\left(1-u_{1} \sigma\right) \beta\left(\frac{S_{1}(t) I_{1}(t)}{N_{1}(t)}-\frac{S_{2}(t) I_{2}(t)}{N_{2}(t)}\right) \\
0 \\
0
\end{array}\right] .
$$

Therefore

$$
\begin{aligned}
\mid F & \left(G_{1}\right)-F\left(G_{2}\right) \mid \\
& =\left|-\left(1-u_{1} \sigma\right) \beta \eta\left(\frac{S_{1}(t) C_{1}(t)}{N_{1}(t)}-\frac{S_{2}(t) C_{2}(t)}{N_{2}(t)}\right)\right| \\
& +\left|-\left(1-u_{1} \sigma\right) \beta \eta_{1}\left(\frac{S_{1}(t) I_{1}(t)}{N_{1}(t)}-\frac{S_{2}(t) I_{2}(t)}{N_{2}(t)}\right)\right| \\
& +\left|\left(1-u_{1} \sigma\right) \beta \eta\left(\frac{S_{1}(t) C_{1}(t)}{N_{1}(t)}-\frac{S_{2}(t) C_{2}(t)}{N_{2}(t)}\right)\right| \\
& +\left|-\left(1-u_{1} \sigma\right) \beta \eta_{1}\left(\frac{S_{1}(t) I_{1}(t)}{N_{1}(t)}-\frac{S_{2}(t) I_{2}(t)}{N_{2}(t)}\right)\right| \\
& \leq 2\left(1-u_{1} \sigma\right) \beta \eta\left|\left(\frac{S_{1}(t) C_{1}(t)}{N_{1}(t)}-\frac{S_{2}(t) C_{2}(t)}{N_{2}(t)}\right)\right|
\end{aligned}
$$

$$
\begin{aligned}
& +2\left(1-u_{1} \sigma\right) \beta \eta_{1}\left|\left(\frac{S_{1}(t) I_{1}(t)}{N_{1}(t)}-\frac{S_{2}(t) I_{2}(t)}{N_{2}(t)}\right)\right| \\
& \leq 2\left(1-u_{1} \sigma\right) \beta \eta\left|S_{1} C_{1}-S_{2} C_{2}\right|+2\left(1-u_{1} \sigma\right) \\
& \cdot \beta \eta_{1}\left|S_{1} I_{1}-S_{2} I_{2}\right| \leq 2\left(1-u_{1} \sigma\right) \\
& \cdot \beta \eta\left|C_{1}\left(S_{1}-S_{2}\right)+S_{2}\left(C_{1}-C_{2}\right)\right|+2\left(1-u_{1} \sigma\right) \\
& \cdot \beta \eta_{1}\left|I_{1}\left(S_{1}-S_{2}\right)+S_{2}\left(I_{1}-I_{2}\right)\right| \leq\left|S_{1}-S_{2}\right| \\
& \cdot\left(2\left(1-u_{1} \sigma\right) \beta \eta\left|C_{1}\right|+2\left(1-u_{1} \sigma\right) \beta \eta_{1}\left|I_{1}\right|\right) \\
& +2\left(1-u_{1} \sigma\right) \beta \eta\left|S_{2}\right|\left|C_{1}-C_{2}\right|+2\left(1-u_{1} \sigma\right) \\
& \cdot \beta \eta_{1}\left|S_{2}\right|\left|I_{1}-I_{2}\right| \leq \frac{2\left(1-u_{1} \sigma\right) \beta \pi}{\mu}(\eta+1)
\end{aligned}
$$




$$
\begin{aligned}
& \cdot\left|S_{1}-S_{2}\right|+\frac{2\left(1-u_{1} \sigma\right) \beta \eta \pi}{\mu}\left|C_{1}-C_{2}\right| \\
& +\frac{2\left(1-u_{1} \sigma\right) \beta \eta_{1} \pi}{\mu}\left|I_{1}-I_{2}\right| \\
& \leq M\left(\left|S_{1}-S_{2}\right|+\left|C_{1}-C_{2}\right|+\left|I_{1}-I_{2}\right|\right),
\end{aligned}
$$

where

$$
\begin{aligned}
M & =\max \left(\frac{2\left(1-u_{1} \sigma\right) \beta \pi}{\mu}(\eta+1), \frac{2\left(1-u_{1} \sigma\right) \beta \eta \pi}{\mu},\right. \\
& \left.\frac{2\left(1-u_{1} \sigma\right) \beta \eta_{1} \pi}{\mu}\right),
\end{aligned}
$$

so that

$$
\begin{aligned}
\left|D\left(G_{1}\right)-D\left(G_{2}\right)\right| \leq & V\left|G_{1}-G_{2}\right|, \\
& \text { where } V=\max (M,\|K\|)<\infty .
\end{aligned}
$$

The function $D$ is therefore uniformly Lipschitz continuous. From the definition of the control measures $u_{1}(t)$ and $u_{2}(t)$ and the constraint on the state variables, such that $S(t)>$ $0, C(t) \geq 0, I(t) \geq 0$, and $R(t) \geq 0$, we observe that a solution of system (31) exists $[40,53,54]$. From the objective functional and its associated constraints in model (2), we can find the optimal solution for our model. Firstly, we find the Lagrangian $(L)$ and Hamiltonian $(H)$ for the control problem [55]. The Lagrangian of the optimal problem is given by

$$
\begin{aligned}
L\left(S, C, I, R, u_{1}, u_{2}\right)= & A_{1} C(t)+A_{2} I(t)+\frac{B_{1}}{2} u_{1}^{2}(t) \\
& +\frac{B_{2}}{2} u_{2}^{2}(t) .
\end{aligned}
$$

Our focus is to find the minimal value of the Lagrangian function, which is done by a pointwise minimization of the Hamiltonian $(H)$ defined as follows (using Pontryagin's maximum principle):

$$
\begin{aligned}
& H\left(S, C, I, R, u_{1}, u_{2}, t\right)=A_{1} C+A_{2} I+\frac{B_{1}}{2} u_{1}^{2}+\frac{B_{2}}{2} u_{2}^{2} \\
& +\lambda_{1}\left[\pi-\frac{\left(1-u_{1} \sigma\right) \beta S\left(\eta C+\eta_{1} I\right)}{N}-\left(\mu+u_{1} \sigma\right) S\right. \\
& +\theta R]+\lambda_{2}\left[\frac{\left(1-u_{1} \sigma\right) \beta S\left(\eta C+\eta_{1} I\right)}{N}\right. \\
& \left.-\left(\alpha+\omega+\mu+u_{2} \gamma\right) C\right]+\lambda_{3}[\alpha C
\end{aligned}
$$

$$
\begin{aligned}
& \left.-\left(\delta+\mu+u_{2} \gamma\right) I\right]+\lambda_{4}\left[u_{2} \gamma I+\left(\omega+u_{2} \gamma\right) C\right. \\
& \left.+u_{1} \sigma S-(\theta+\mu) R\right]
\end{aligned}
$$

where $\lambda_{i}, i=1,2,3,4$, are the adjoint variables associated with $S(t), C(t), I(t)$, and $R(t)$, defined by

$$
\begin{aligned}
& \frac{d \lambda_{1}}{d t}=-\frac{\partial H}{\partial S} \\
& \frac{d \lambda_{2}}{d t}=-\frac{\partial H}{\partial C} \\
& \frac{d \lambda_{3}}{d t}=-\frac{\partial H}{\partial I} \\
& \frac{d \lambda_{4}}{d t}=-\frac{\partial H}{\partial R} .
\end{aligned}
$$

Theorem 6. There exists an optimal control pair $u_{1}^{*}(t), u_{2}^{*}(t)$ such that

$$
J\left(u_{1}^{*}(t), u_{2}^{*}(t)\right)=\min _{u_{1}, u_{2} \in U} J\left(u_{1}(t), u_{2}(t)\right) .
$$

Proof. We start our proof by considering the properties of the existence of the optimal control (see [56]). Following [57], the set of control measures with corresponding state variables are positive. The set $U$ is convex and closed by definition. Therefore, our optimal system is closed and bounded which ascertains the compactness required for the existence of the optimal control. Additionally, the integrand in the objective functional (29), $A_{1} C(t)+A_{2} I(t)+\left(B_{1} / 2\right) u_{1}^{2}(t)+\left(B_{2} / 2\right) u_{2}^{2}(t)$, is convex on the control set $U$. Furthermore, we can state that there exists a positive constant $\rho>1$ [58], and nonnegative numbers $v_{1}$ and $v_{2}$ such that the objective functional has a lower bound of $\nu_{1}\left(\left|u_{1}\right|^{2}+\left|u_{2}\right|^{2}\right)^{\rho / 2}-v_{2}$ so that

$$
J\left(u_{1}(t), u_{2}(t)\right) \geq v_{1}\left(\left|u_{1}\right|^{2}+\left|u_{2}\right|^{2}\right)^{\rho / 2}-v_{2},
$$

since the control measures and the state variables are bounded, this leads us to a compact proof of existence of the optimal control.

3.2. Characterization of the Optimal Control. We will apply Pontryagin's maximum principle to the Hamiltonian function above to derive the necessary condition of optimality for our control problem. 
Theorem 7. Let $S, C, I$, and $R$ be optimal state solutions with corresponding optimal control variables $u_{1}^{*}$ and $u_{2}^{*}$ for the objective functional and its constraints in model (2) with $N=S+C+I+R$. Then, there exist four adjoint variables $\lambda_{1}$, $\lambda_{2}, \lambda_{3}$, and $\lambda_{4}$ that satisfy

$$
\begin{aligned}
& \frac{d \lambda_{1}}{d t}=\lambda_{1}\left[\frac{\left(1-u_{1} \sigma\right) \beta\left(\eta C+\eta_{1} I\right)}{N}\right. \\
& \left.-\frac{\left(1-u_{1} \sigma\right) \beta S\left(\eta C+\eta_{1} I\right)}{N^{2}}+\left(\mu+u_{1} \sigma\right)\right] \\
& +\lambda_{2}\left[\frac{\left(1-u_{1} \sigma\right) \beta S\left(\eta C+\eta_{1} I\right)}{N^{2}}\right. \\
& \left.-\frac{\left(1-u_{1} \sigma\right) \beta\left(\eta C+\eta_{1} I\right)}{N}\right]-\lambda_{4} u_{1} \sigma, \\
& \frac{d \lambda_{2}}{d t}=-A_{1}+\lambda_{1}\left[\frac{\left(1-u_{1} \sigma\right) \beta \eta S}{N}\right. \\
& -\frac{\left.\left(1-u_{1} \sigma\right) \beta S\left(\eta C+\eta_{1} I\right)\right]+\lambda_{2}(\alpha+\omega+\mu}{N^{2}} \\
& \left.+u_{2} \gamma\right)+\lambda_{2}\left[\frac{\left(1-u_{1} \sigma\right) \beta S\left(\eta C+\eta_{1} I\right)}{N^{2}}\right. \\
& -\frac{\left.\left(1-u_{1} \sigma\right) \beta \eta S\right]-\lambda_{3} \alpha-\lambda_{4}\left(\omega+u_{2} \gamma\right),}{N}
\end{aligned}
$$

$$
\begin{aligned}
\frac{d \lambda_{3}}{d t} & =-A_{2}+\lambda_{1}\left[\frac{\left(1-u_{1} \sigma\right) \beta \eta_{1} S}{N}\right. \\
& \left.-\frac{\left(1-u_{1} \sigma\right) \beta S\left(\eta C+\eta_{1} I\right)}{N^{2}}\right] \\
& +\lambda_{2}\left[\frac{\left(1-u_{1} \sigma\right) \beta S\left(\eta C+\eta_{1} I\right)}{N^{2}}\right. \\
& \left.-\frac{\left(1-u_{1} \sigma\right) \beta \eta_{1} S}{N}\right]+\lambda_{3}\left(\delta+\mu+u_{2} \gamma\right)-\lambda_{4} u_{2} \gamma,
\end{aligned}
$$$$
\frac{d \lambda_{4}}{d t}=-\lambda_{1} \theta+\lambda_{4}(\theta+\mu)
$$

with transversality conditions

$$
\lambda_{i}(T)=0, \quad i=1,2,3,4
$$

for the control set $U=\left(u_{1}, u_{2}\right)$, such that $\frac{\partial H}{\partial u_{i}}=0$,

where $i=1,2$.

Therefore, the optimal control pair $\left(u_{1}^{*}, u_{2}^{*}\right)$ is given by

$$
\begin{aligned}
& u_{1}^{*}=\min \left\{\max \left(0, \frac{1}{B_{1}}\left[\frac{\beta S\left(\eta C+\eta_{1} I\right)}{(S+C+I+R)}\left(\lambda_{1}-\lambda_{2}\right)+\left(\lambda_{1}-\lambda_{4}\right) \sigma S\right]\right), u_{1 \max }\right\}, \\
& u_{2}^{*}=\min \left\{\max \left(0, \frac{\gamma\left(\lambda_{2}-\lambda_{4}\right) C+\gamma\left(\lambda_{3}-\lambda_{4}\right) I}{B_{2}}\right), u_{2 \max }\right\} .
\end{aligned}
$$

Proof. We use the Hamiltonian function in (38) in order to obtain the adjoint relations and the transversality conditions. We set the state variables in the Hamiltonian function to $S, C, I$, and $R$, and differentiating the Hamiltonian $(H)$ with respect to $S, C, I$, and $R$, respectively, yields (42). Also, differentiating the Hamiltonian $(H)$ with respect to the control measures $u_{1} \sigma$ and $u_{2}$ in the interior of $U$, we obtain the optimality conditions below:

$$
\begin{aligned}
\frac{\partial H}{\partial u_{1}}= & B_{1} u_{1}-\lambda_{1} \frac{\beta S\left(\eta C+\eta_{1} I\right)}{S+C+I+R}-\lambda_{1} \sigma S \\
& +\lambda_{2} \frac{\beta S\left(\eta C+\eta_{1} I\right)}{S+C+I+R}+\lambda_{4} \sigma S=0, \\
\frac{\partial H}{\partial u_{2}}= & B_{2} u_{2}-\lambda_{2} \gamma C-\lambda_{3} \gamma I+\lambda_{4} \gamma C+\lambda_{4} \gamma I=0 .
\end{aligned}
$$

Plugging $u_{1}=u_{1}^{*}$ and $u_{2}=u_{2}^{*}$ into (45) and solving the optimal control pair $\left(u_{1}^{*}, u_{2}^{*}\right)$, we have

$$
\begin{aligned}
& u_{1}^{*} \\
& =\frac{1}{B_{1}}\left[\frac{\beta S\left(\eta C+\eta_{1} I\right)}{(S+C+I+R)}\left(\lambda_{1}-\lambda_{2}\right)+\left(\lambda_{1}-\lambda_{4}\right) \sigma S\right], \\
& u_{2}^{*}=\frac{\gamma\left(\lambda_{2}-\lambda_{4}\right) C+\gamma\left(\lambda_{3}-\lambda_{4}\right) I}{B_{2}} .
\end{aligned}
$$

The two control measures which are bounded with lower bounds zero and upper bounds $u_{i \max }=1$, where $i=1,2$, give 


$$
\begin{aligned}
& u_{1}^{*} \in U \Longrightarrow \\
& u_{1}^{*}(t) \\
& \quad= \begin{cases}0 & \text { if } \frac{1}{B_{1}}\left[\frac{\beta S\left(\eta C+\eta_{1} I\right)}{(S+C+I+R)}\left(\lambda_{1}-\lambda_{2}\right)+\left(\lambda_{1}-\lambda_{4}\right) \sigma S\right] \leq 0, \\
\frac{1}{B_{1}}\left[\frac{\beta S\left(\eta C+\eta_{1} I\right)}{(S+C+I+R)}\left(\lambda_{1}-\lambda_{2}\right)+\left(\lambda_{1}-\lambda_{4}\right) \sigma S\right] & \text { if } 0<\frac{1}{B_{1}}\left[\frac{\beta S\left(\eta C+\eta_{1} I\right)}{(S+C+I+R)}\left(\lambda_{1}-\lambda_{2}\right)+\left(\lambda_{1}-\lambda_{4}\right) \sigma S\right]<u_{1 \max }, \\
u_{1 \max } & \text { if } \frac{1}{B_{1}}\left[\frac{\beta S\left(\eta C+\eta_{1} I\right)}{(S+C+I+R)}\left(\lambda_{1}-\lambda_{2}\right)+\left(\lambda_{1}-\lambda_{4}\right) \sigma S\right] \geq u_{1 \max },\end{cases}
\end{aligned}
$$

$u_{2}^{*} \in U \Longrightarrow$

$$
u_{2}^{*}(t)= \begin{cases}0 & \text { if } \frac{\gamma\left(\lambda_{2}-\lambda_{4}\right) C+\gamma\left(\lambda_{3}-\lambda_{4}\right) I}{B_{2}} \leq 0, \\ \frac{\gamma\left(\lambda_{2}-\lambda_{4}\right) C+\gamma\left(\lambda_{3}-\lambda_{4}\right) I}{B_{2}} & \text { if } 0<\frac{\gamma\left(\lambda_{2}-\lambda_{4}\right) C+\gamma\left(\lambda_{3}-\lambda_{4}\right) I}{B_{2}}<u_{2 \max }, \\ u_{2 \max } & \text { if } \frac{\gamma\left(\lambda_{2}-\lambda_{4}\right) C+\gamma\left(\lambda_{3}-\lambda_{4}\right) I}{B_{2}} \geq u_{2 \max } .\end{cases}
$$

Using (47), the optimal control measures are characterized as (44), completing the proof.
Therefore, our optimality system is given by

$$
\begin{aligned}
& \frac{d S}{d t}=\pi-\left(1-\min \left\{\max \left(0, \frac{1}{B_{1}}\left[\frac{\beta S\left(\eta_{1} I+\eta C\right)}{(S+C+I+R)}\left(\lambda_{1}-\lambda_{2}\right)+\left(\lambda_{1}-\lambda_{4}\right) \sigma S\right]\right), u_{1 \max }\right\}\right) \frac{\beta S\left(\eta C+\eta_{1} I\right)}{N} \\
& -\left(\mu+\left(\min \left\{\max \left(0, \frac{1}{B_{1}}\left[\frac{\beta S\left(\eta C+\eta_{1} I\right)}{(S+C+I+R)}\left(\lambda_{1}-\lambda_{2}\right)+\left(\lambda_{1}-\lambda_{4}\right) \sigma S\right]\right), u_{1 \max }\right\}\right) \sigma\right) S+\theta R, \\
& \frac{d C}{d t}=\left(1-\min \left\{\max \left(0, \frac{1}{B_{1}}\left[\frac{\beta S\left(\eta C+\eta_{1} I\right)}{(S+C+I+R)}\left(\lambda_{1}-\lambda_{2}\right)+\left(\lambda_{1}-\lambda_{4}\right) \sigma S\right]\right), u_{1 \max }\right\}\right) \frac{\beta S\left(\eta C+\eta_{1} I\right)}{N} \\
& -\left(\alpha+\omega+\mu+\left(\min \left\{\max \left(0, \frac{\gamma\left(\lambda_{2}-\lambda_{4}\right) C+\gamma\left(\lambda_{3}-\lambda_{4}\right) I}{B_{2}}\right), u_{2 \max }\right\}\right) \gamma\right) C, \\
& \frac{d I}{d t}=\alpha C-\left(\delta+\mu+\left(\min \left\{\max \left(0, \frac{\gamma\left(\lambda_{2}-\lambda_{4}\right) C+\gamma\left(\lambda_{3}-\lambda_{4}\right) I}{B_{2}}\right), u_{2 \max }\right\}\right) \gamma\right) I, \\
& \frac{d R}{d t}=\left(\left(\min \left\{\max \left(0, \frac{\gamma\left(\lambda_{2}-\lambda_{4}\right) C+\gamma\left(\lambda_{3}-\lambda_{4}\right) I}{B_{2}}\right), u_{2 \max }\right\}\right) \gamma\right) I \\
& +\left(\omega+\left(\min \left\{\max \left(0, \frac{\gamma\left(\lambda_{2}-\lambda_{4}\right) C+\gamma\left(\lambda_{3}-\lambda_{4}\right) I}{B_{2}}\right), u_{2 \max }\right\}\right) \gamma\right) C-(\theta+\mu) R \\
& +\left(\min \left\{\max \left(0, \frac{1}{B_{1}}\left[\frac{\beta S\left(\eta C+\eta_{1} I\right)}{(S+C+I+R)}\left(\lambda_{1}-\lambda_{2}\right)+\left(\lambda_{1}-\lambda_{4}\right) \sigma S\right]\right), u_{1 \max }\right\}\right) \sigma S \\
& \frac{d \lambda_{1}}{d t}=\lambda_{1}\left[\left(1-\left(\min \left\{\max \left(0, \frac{1}{B_{1}}\left[\frac{\beta S\left(\eta C+\eta_{1} I\right)}{(S+C+I+R)}\left(\lambda_{1}-\lambda_{2}\right)+\left(\lambda_{1}-\lambda_{4}\right) \sigma S\right]\right), u_{1 \max }\right\}\right)\right) \frac{\beta\left(\eta C+\eta_{1} I\right)}{N}\right] \\
& -\lambda_{1}\left[\left(1-\left(\min \left\{\max \left(0, \frac{1}{B_{1}}\left[\frac{\beta S\left(\eta C+\eta_{1} I\right)}{(S+C+I+R)}\left(\lambda_{1}-\lambda_{2}\right)+\left(\lambda_{1}-\lambda_{4}\right) \sigma S\right]\right), u_{1 \max }\right\}\right)\right) \frac{\beta S\left(\eta C+\eta_{1} I\right)}{N^{2}}\right]
\end{aligned}
$$




$$
\begin{aligned}
& +\lambda_{1}\left[\left(\mu+\left(\min \left\{\max \left(0, \frac{1}{B_{1}}\left[\frac{\beta S\left(\eta C+\eta_{1} I\right)}{(S+C+I+R)}\left(\lambda_{1}-\lambda_{2}\right)+\left(\lambda_{1}-\lambda_{4}\right) \sigma S\right]\right), u_{1 \max }\right\}\right) \sigma\right)\right] \\
& +\lambda_{2}\left[\left(1-\left(\min \left\{\max \left(0, \frac{1}{B_{1}}\left[\frac{\beta S\left(\eta C+\eta_{1} I\right)}{(S+C+I+R)}\left(\lambda_{1}-\lambda_{2}\right)+\left(\lambda_{1}-\lambda_{4}\right) \sigma S\right]\right), u_{1 \max }\right\}\right)\right) \frac{\beta S\left(\eta C+\eta_{1} I\right)}{N^{2}}\right] \\
& -\lambda_{2}\left[\left(1-\left(\min \left\{\max \left(0, \frac{1}{B_{1}}\left[\frac{\beta S\left(\eta C+\eta_{1} I\right)}{(S+C+I+R)}\left(\lambda_{1}-\lambda_{2}\right)+\left(\lambda_{1}-\lambda_{4}\right) \sigma S\right]\right), u_{1 \max }\right\}\right)\right) \frac{\beta\left(\eta C+\eta_{1} I\right)}{N}\right] \\
& -\lambda_{4}\left(\min \left\{\max \left(0, \frac{1}{B_{1}}\left[\frac{\beta S\left(\eta C+\eta_{1} I\right)}{(S+C+I+R)}\left(\lambda_{1}-\lambda_{2}\right)+\left(\lambda_{1}-\lambda_{4}\right) \sigma S\right]\right), u_{1 \max }\right\}\right) \sigma, \\
& \frac{d \lambda_{2}}{d t}=-A_{1}+\lambda_{1}\left[\left(1-\left(\min \left\{\max \left(0, \frac{1}{B_{1}}\left[\frac{\beta S\left(\eta C+\eta_{1} I\right)}{(S+C+I+R)}\left(\lambda_{1}-\lambda_{2}\right)+\left(\lambda_{1}-\lambda_{4}\right) \sigma S\right]\right), u_{1 \max }\right\}\right)\right) \frac{\beta \eta S}{N}\right] \\
& -\lambda_{1}\left[\left(1-\left(\min \left\{\max \left(0, \frac{1}{B_{1}}\left[\frac{\beta S\left(\eta C+\eta_{1} I\right)}{(S+C+I+R)}\left(\lambda_{1}-\lambda_{2}\right)+\left(\lambda_{1}-\lambda_{4}\right) \sigma S\right]\right), u_{1 \max }\right\}\right)\right) \frac{\beta S\left(\eta C+\eta_{1} I\right)}{N^{2}}\right] \\
& +\lambda_{2}\left(\alpha+\omega+\mu+\left(\min \left\{\max \left(0, \frac{\gamma\left(\lambda_{2}-\lambda_{4}\right) C+\gamma\left(\lambda_{3}-\lambda_{4}\right) I}{B_{2}}\right), u_{2 \max }\right\}\right) \gamma\right) \\
& +\lambda_{2}\left[\left(1-\left(\min \left\{\max \left(0, \frac{1}{B_{1}}\left[\frac{\beta S\left(\eta C+\eta_{1} I\right)}{(S+C+I+R)}\left(\lambda_{1}-\lambda_{2}\right)+\left(\lambda_{1}-\lambda_{4}\right) \sigma S\right]\right), u_{1 \max }\right\}\right)\right) \frac{\beta S\left(\eta C+\eta_{1} I\right)}{N^{2}}\right] \\
& -\lambda_{2}\left[\left(1-\left(\min \left\{\max \left(0, \frac{1}{B_{1}}\left[\frac{\beta S\left(\eta C+\eta_{1} I\right)}{(S+C+I+R)}\left(\lambda_{1}-\lambda_{2}\right)+\left(\lambda_{1}-\lambda_{4}\right) \sigma S\right]\right), u_{1 \max }\right\}\right)\right) \frac{\beta \eta S}{N}\right]-\lambda_{3} \alpha \\
& -\lambda_{4}\left(\omega+\left(\min \left\{\max \left(0, \frac{\gamma\left(\lambda_{2}-\lambda_{4}\right) C+\gamma\left(\lambda_{3}-\lambda_{4}\right) I}{B_{2}}\right), u_{2 \max }\right\}\right) \gamma\right) \\
& \frac{d \lambda_{3}}{d t}=-A_{2}+\lambda_{1}\left[\left(1-\left(\min \left\{\max \left(0, \frac{1}{B_{1}}\left[\frac{\beta S\left(\eta C+\eta_{1} I\right)}{(S+C+I+R)}\left(\lambda_{1}-\lambda_{2}\right)+\left(\lambda_{1}-\lambda_{4}\right) \sigma S\right]\right), u_{1 \max }\right\}\right)\right) \frac{\beta \eta_{1} S}{N}\right] \\
& -\lambda_{1}\left[\left(1-\left(\min \left\{\max \left(0, \frac{1}{B_{1}}\left[\frac{\beta S\left(\eta C+\eta_{1} I\right)}{(S+C+I+R)}\left(\lambda_{1}-\lambda_{2}\right)+\left(\lambda_{1}-\lambda_{4}\right) \sigma S\right]\right), u_{1 \max }\right\}\right)\right) \frac{\beta\left(\eta C+\eta_{1} I\right)}{N^{2}}\right] \\
& +\lambda_{2}\left[\left(1-\left(\min \left\{\max \left(0, \frac{1}{B_{1}}\left[\frac{\beta S\left(\eta C+\eta_{1} I\right)}{(S+C+I+R)}\left(\lambda_{1}-\lambda_{2}\right)+\left(\lambda_{1}-\lambda_{4}\right) \sigma S\right]\right), u_{1 \max }\right\}\right)\right) \frac{\beta\left(\eta C+\eta_{1} I\right)}{N^{2}}\right] \\
& -\lambda_{2}\left[\left(1-\left(\min \left\{\max \left(0, \frac{1}{B_{1}}\left[\frac{\beta S\left(\eta C+\eta_{1} I\right)}{(S+C+I+R)}\left(\lambda_{1}-\lambda_{2}\right)+\left(\lambda_{1}-\lambda_{4}\right) \sigma S\right]\right), u_{1 \max }\right\}\right)\right) \frac{\beta \eta_{1} S}{N}\right] \\
& +\lambda_{3}\left(\delta+\mu+\left(\min \left\{\max \left(0, \frac{\gamma\left(\lambda_{2}-\lambda_{4}\right) C+\gamma\left(\lambda_{3}-\lambda_{4}\right) I}{B_{2}}\right), u_{2 \max }\right\}\right) \gamma\right) \\
& -\lambda_{4}\left(\min \left\{\max \left(0, \frac{\gamma\left(\lambda_{2}-\lambda_{4}\right) C+\gamma\left(\lambda_{3}-\lambda_{4}\right) I}{B_{2}}\right), u_{2 \max }\right\}\right) \gamma \\
& \frac{d \lambda_{4}}{d t}=-\lambda_{1} \theta+\lambda_{4}(\theta+\mu) \text {, }
\end{aligned}
$$

with $\lambda_{i}(T)=0, i=1,2,3,4$

\section{Numerical Simulations of the Optimal Control}

The optimality system consisting of the state equations (2) and the adjoint equations (45) is solved using the forwardbackward sweep scheme. The solution is started with an initial guess for the control measures and the final time set to $T=30$ days and later varied to $T=60$ days and $T=80$ days. The state system is solved forward in time while the costate system is solved backward in time. The current solutions of the state system together with the initial guess for the control measures $u_{1}$ and $u_{2}$ are used to solve the costate system. The controls $u_{1}$ 


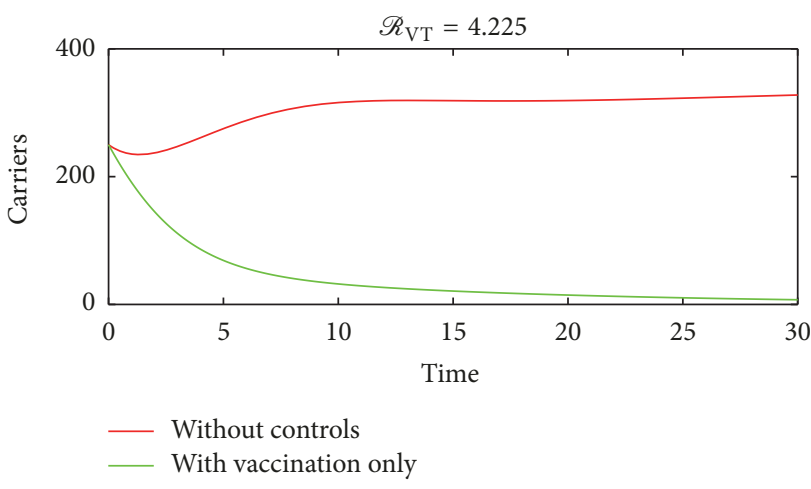

(a) The optimal effect on $C(t)$ with vaccination control

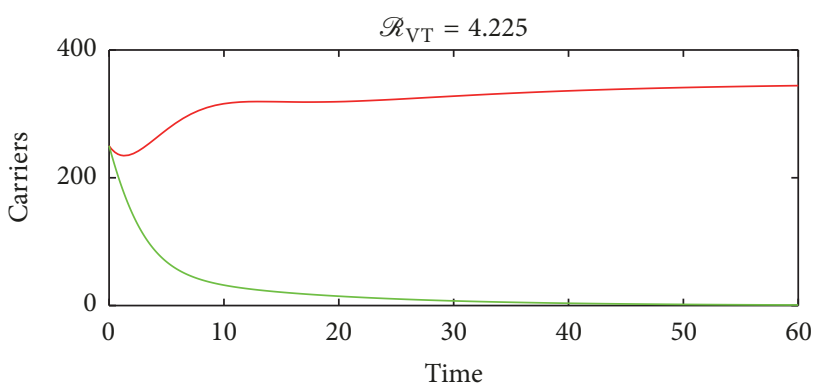

Without controls

- With vaccination only

(c) The optimal effect on $C(t)$ with an increase in time

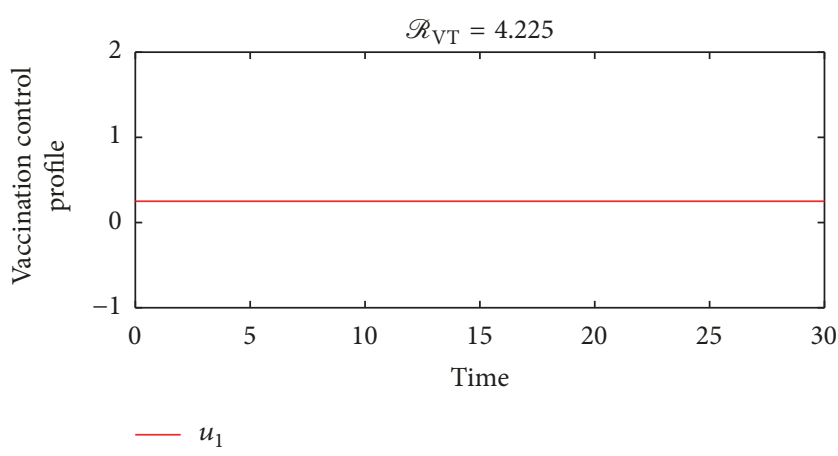

(e) Vaccination control profile

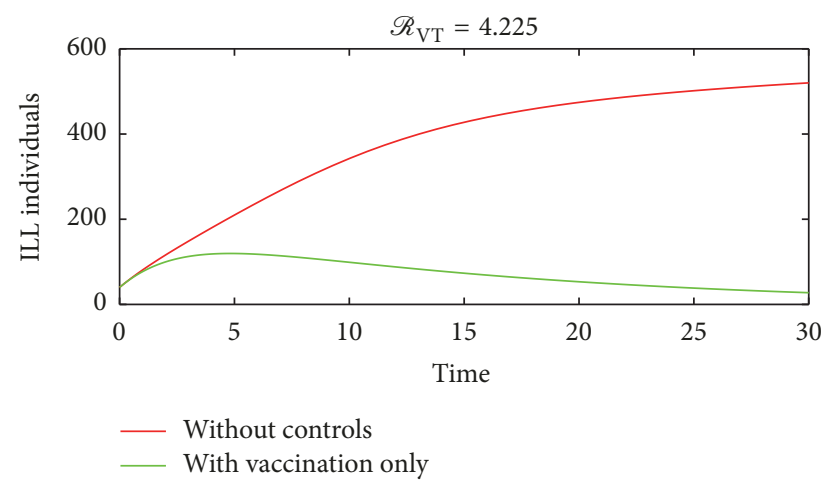

(b) The optimal effect on $I(t)$ with vaccination control

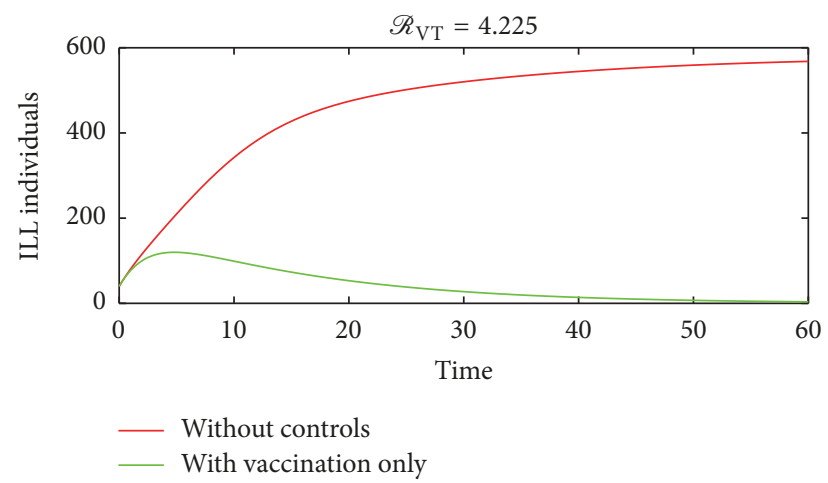

(d) The optimal effect on $I(t)$ with an increase in time

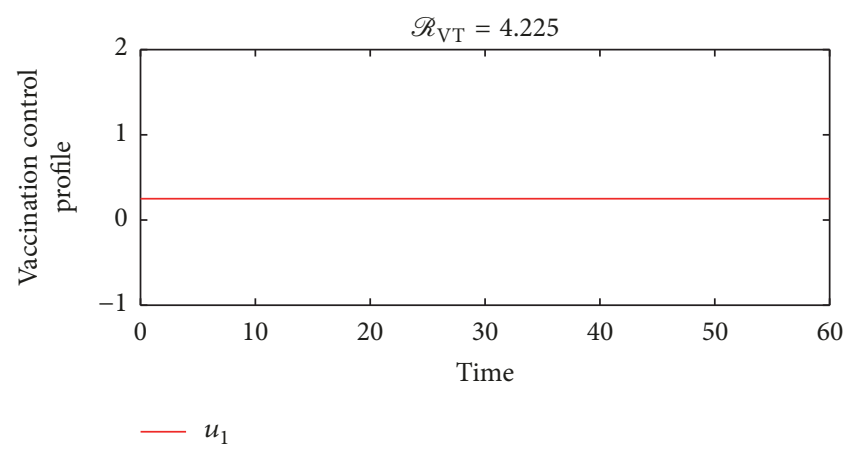

(f) Optimal control profile with an increase in time

FiguRE 4: (a), (b), (c), and (d) show that administering vaccination on susceptible individuals reduces the number of secondary infections in the carrier and the ill population. (e) and (f) show the optimal control profile of vaccination. Initial conditions $S(0)=700, C(0)=$ 250, $I(0)=40$, and $R(0)=10$ and the parameter values $\pi=100, \eta=0.35, \omega=0.2, \delta=0.1, \sigma=1, \alpha=0.2$ and $\theta=0.0839$ were used in this simulation.

and $u_{2}$ are updated using the characterizations in (47). With the current cosystem solution and updated controls, the state system is solved and the whole solution process continues until convergence is achieved. Since bacterial meningitis is an endemic disease especially in Sub-Sahara Africa, parameter values that make effective reproduction number $\mathscr{R}_{\mathrm{VT}}$ more than unity are considered. Taking the associated costs on carriers and ill individuals as $A_{1}=A_{2}=1, B_{1}=2$, and $B_{2}=4$, the graphical results of the model are as follows. Figures 4 and 5, show the dynamics of the infection in the presences of control measures and without control measures in a more localised form. Figure 5(f) shows the effect of varying the cost associated with the treatment control. It is seen that if the weight of treatment cost $B_{2}$ is continuously increased, the upper bound time reduces to about 9 days as indicated by the red line in Figure 5(f). This is true because a higher cost of treatment causes the use of the treatment control to be less. Figure 6 shows the spreading rate of meningitis with and without control measures in a highly dense population, thus in colleges, prisons, cities, and so forth, which suggests that when carriers become more in a highly populated settings there is a likelihood of the infection spreading faster, thereby leading to a corresponding increase in the number of ill (sick) individuals; hence the trajectories in Figures 6(a) and 6(b) show that there is a significant difference in the number of carriers and infected 


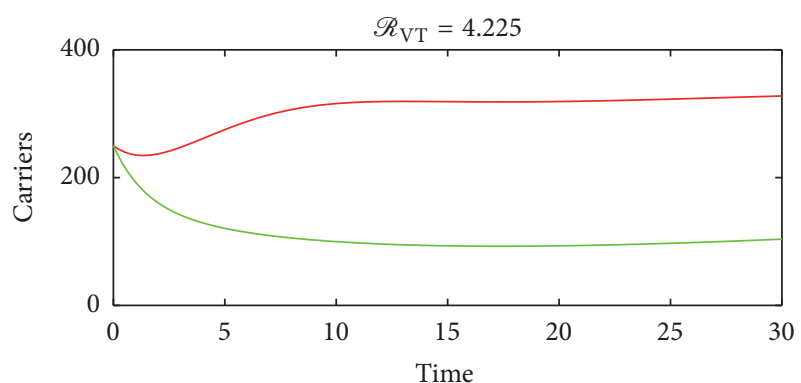

- Without controls

_ With treatment only

(a) The optimal effect on $C(t)$ with treatment control

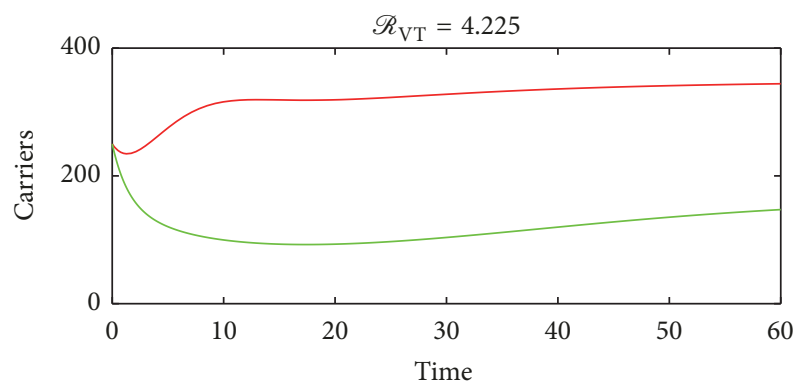

Without controls

_ With treatment only

(c) The optimal effect on $C(t)$ with an increase in time

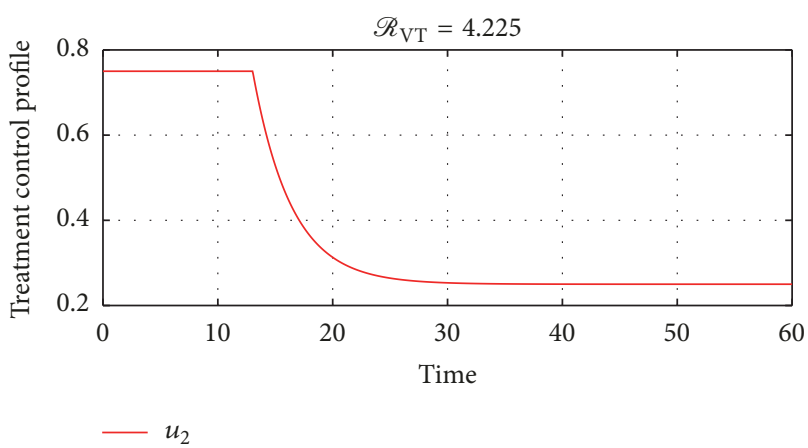

(e) Treatment control effect

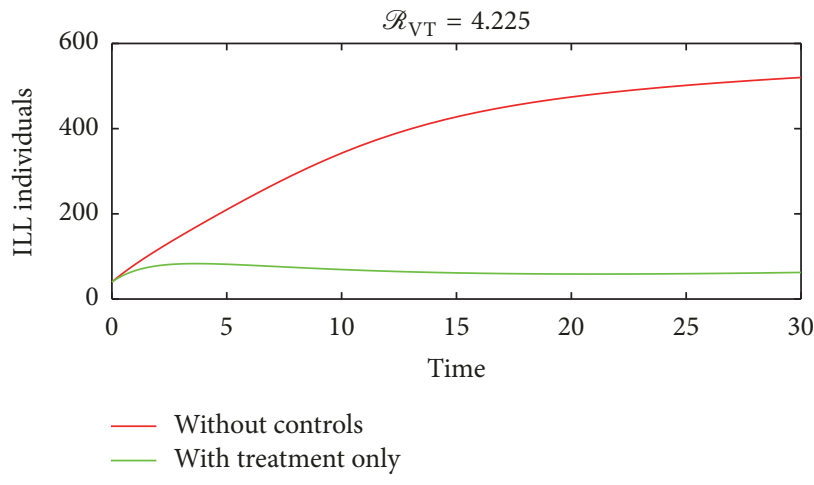

(b) The optimal effect on $I(t)$ with treatment control

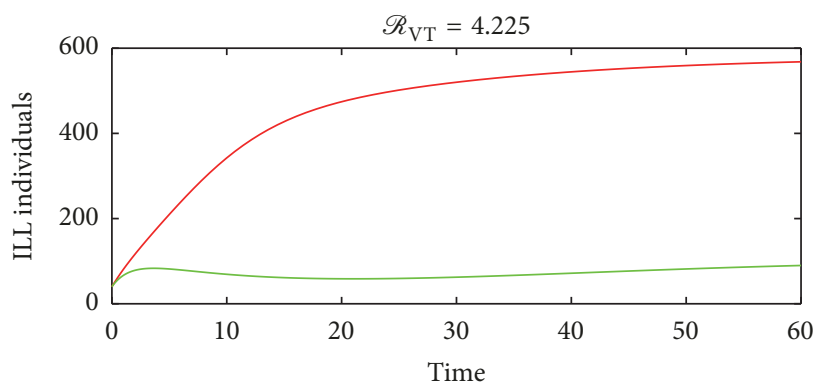

Without controls

_ With treatment only

(d) The optimal effect on $I(t)$ with an increase in time

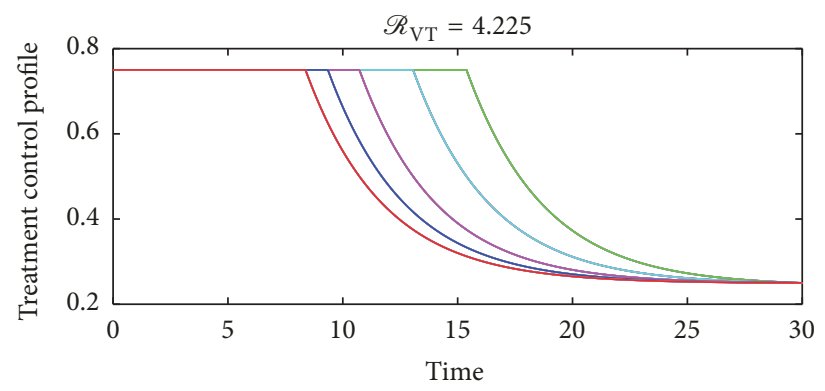

$$
\begin{aligned}
A_{1} & =A_{2}=1 B_{1}=2 B_{2}=16 \\
A_{1} & =A_{2}=1 B_{1}=2 B_{2}=12 \\
A_{1} & =A_{2}=1 B_{1}=2 B_{2}=8 \\
A_{1} & =A_{2}=1 B_{1}=2 B_{2}=4 \\
A_{1}=A_{2} & =1 B_{1}=2 B_{2}=2
\end{aligned}
$$

(f) Optimal control plot of treatment with different cost weights

FIGURE 5: (a) and (b) show that giving treatment to carriers and the ill individuals reduces the number of secondary infections in the carrier and the ill population but the disease may not be eradicated if the use of only treatment is stuck to. Furthermore, we increased time so as to see whether the disease could be eradicated when using treatment only. We see in (c) and (d) that there is a possibility of recurrence of the disease using only treatment control strategy as indicated by the curved green line in (c) and the linear flow of the green line in (d). (e) shows that, to minimize bacterial meningitis outbreak in 30 days, the treatment control should be held intensively for 30 days at a constant rate. (f) shows the optimal control plot of treatment with different cost weights.

individuals with and without control measures. Therefore, applying both vaccination and treatment has a higher rate of controlling bacterial meningitis than depending only on one control method. Figures 6(a) and 6(b) also show that as infections increase in the population, there is a need for introducing vaccination and treatment at the earlier stage so as to minimize the spread of the infection in the population.
This further suggests that college students and prisoners should be given regular vaccination against meningitis, since bacterial meningitis is more likely to affect college students and prisoners than other people, due to the close proximate of beds in the dormitories and prison cells, especially in Sub-Saharan Africa. Figure 6(c) shows that the trajectory for treatment moves to an upper bound at about $t=35$ days and 


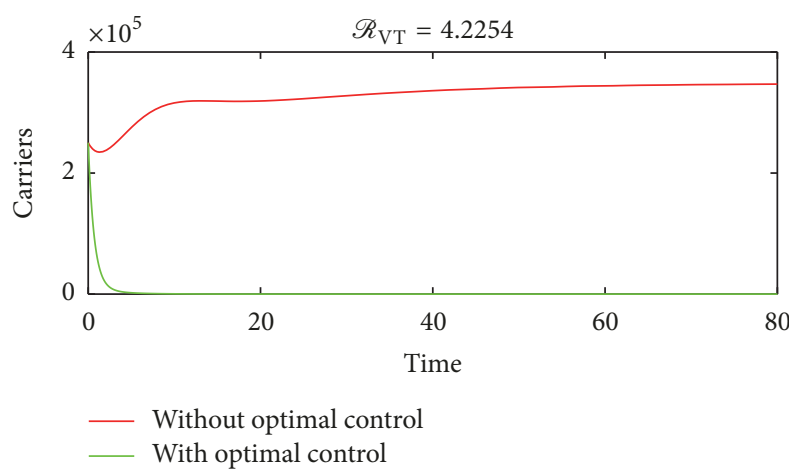

(a) The optimal effect on $C(t)$ with both control measures

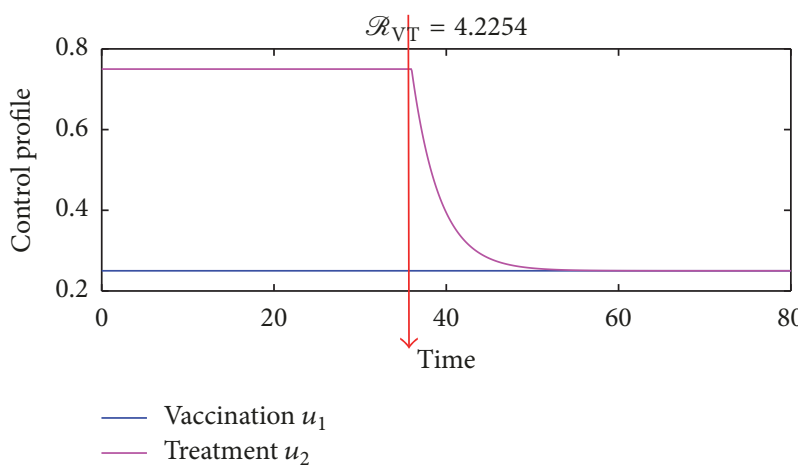

(c) Simulation diagram for control measures

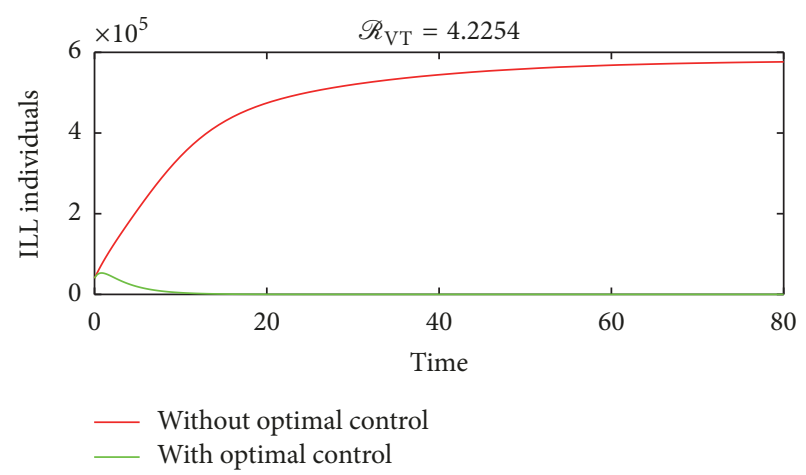

(b) The optimal effect on $I(t)$ with both control measures

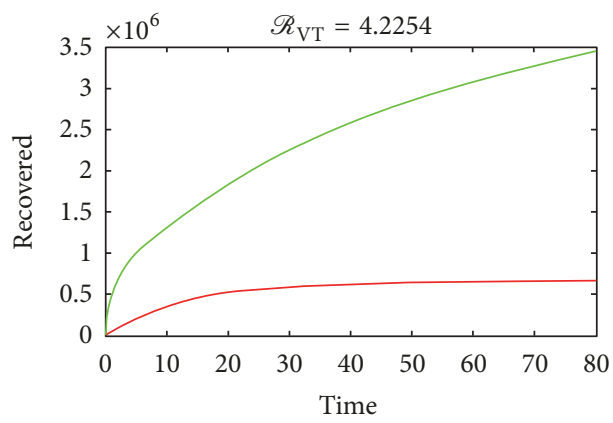

- Without optimal control

_ With optimal control

(d) Immunity of recovered individuals

FIGURE 6: Further simulation trajectories. (d) shows the immunity levels when the two control measures are applied with the following parameter values: $\pi=100000, \eta=0.35, \omega=0.2, \delta=0.1, \sigma=1, \alpha=0.2$, and $\theta=0.0839$.

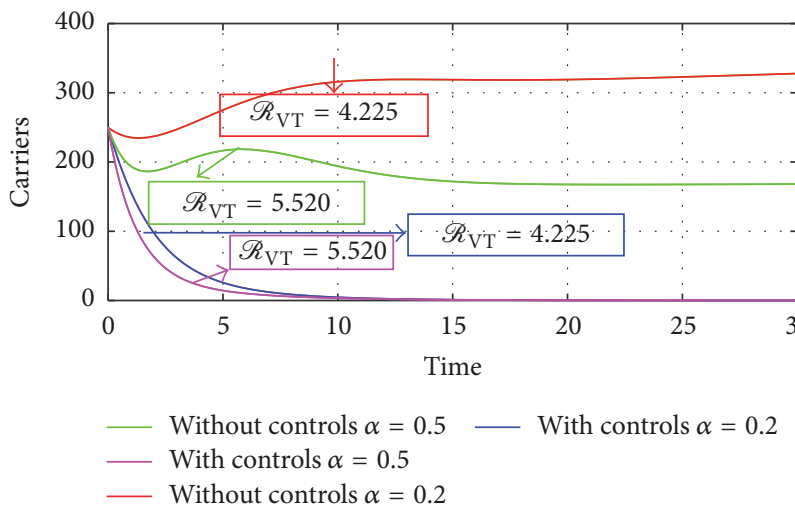

(a) Optimal simulation effect in varying $\alpha$ on $C(t)$

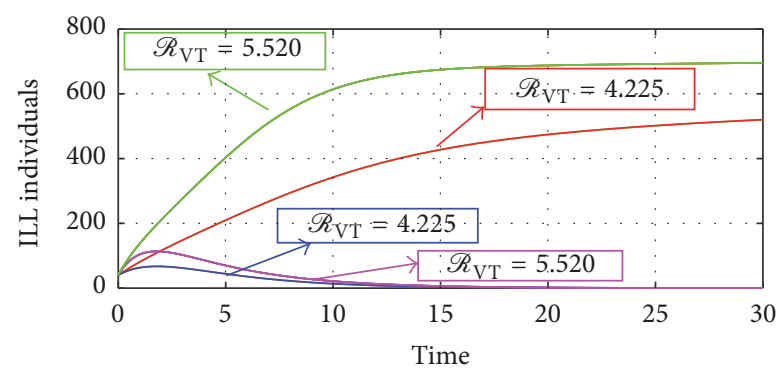

Without controls $\alpha=0.5 \quad$ With controls $\alpha=0.2$
With controls $\alpha=0.5$
Without controls $\alpha=0.2$

(b) Optimal simulation effect in varying $\alpha$ on $I(t)$

FIGURE 7: Simulation results of the optimal control model with $\alpha=0.2-0.5$, on carriers and the ill compartment.

slowly decreases to a lower bound, which implies that, during the period before 35 days, substantial amount of the control $u_{2}$ should be applied while administering the vaccination control in the susceptible population so as to reduce the number of carriers and ill individuals until the 50th day. Afterwards, less amount of treatment can be used since the number of carriers and ill individuals will be considerably reduced by the earlier investment in treatment within the period before 35 days.
Figures $7(a), 7(b), 8(c)$, and $8(d)$ show the impact of varying $\alpha$ and $\eta$. It can be seen that an increase in $\alpha$ and $\eta$ without control measures has a corresponding decrease in the number of carriers and a considerable increase in the number of infected individuals. Therefore, applying the control measures gives a drastic decrease in the number of infections in both carriers and the ill compartment at a higher rate of $\alpha=0.5$ and $\eta=0.65$. Furthermore, in Figures 7(a) and $7(b)$, authors kept the natural recovery rate $(\omega)$ fixed at 


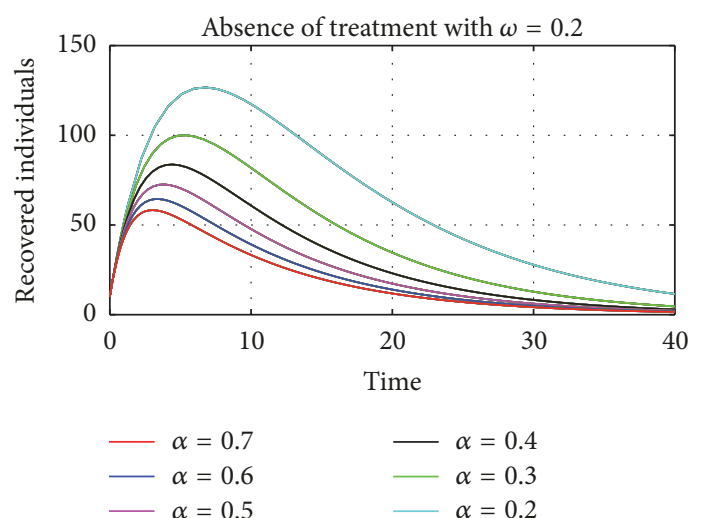

(a) Numerical simulation without control measures on $R(t)$

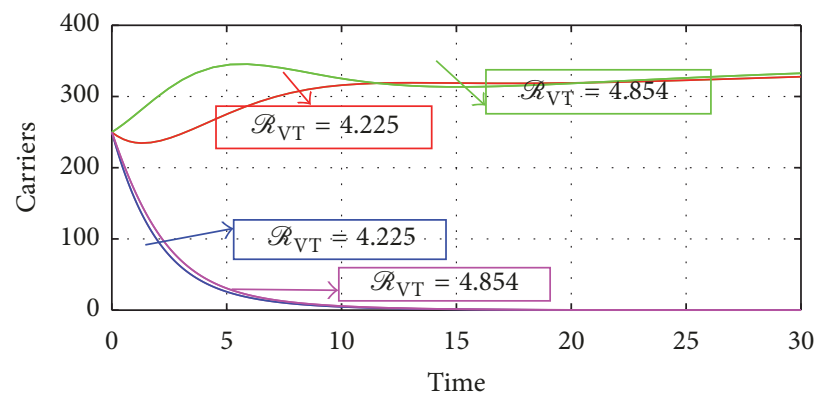

Without controls $\eta=0.65$ With controls $\eta=0.35$

With controls $\eta=0.65$

Without controls $\eta=0.35$

(c) Optimal simulation effect in varying $\eta$ on $C(t)$

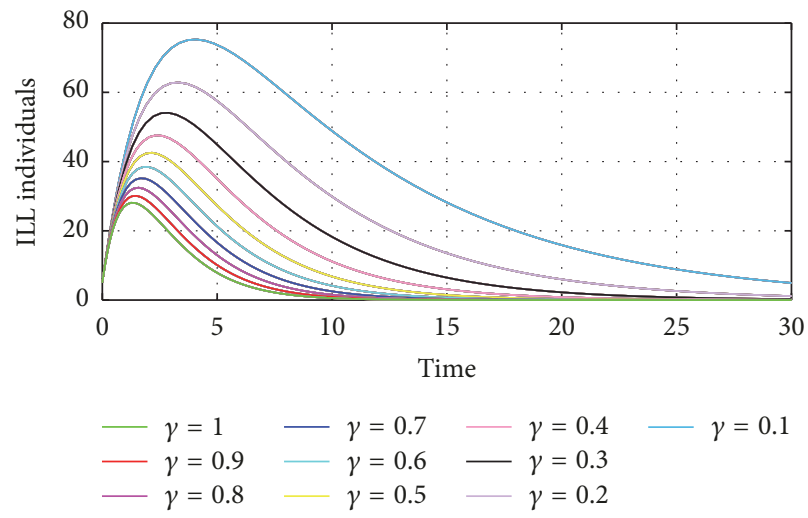

(e) Effectiveness of treatment $\gamma$ on $I(t)$

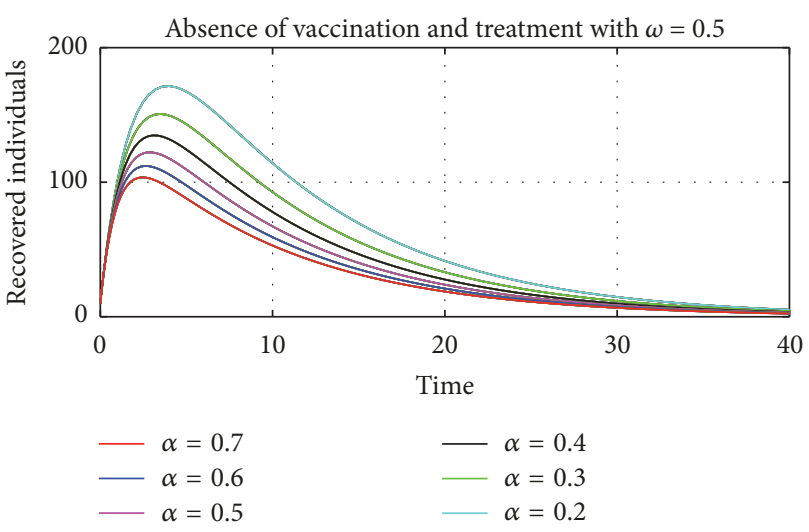

(b) Numerical simulation without control measures on $R(t)$

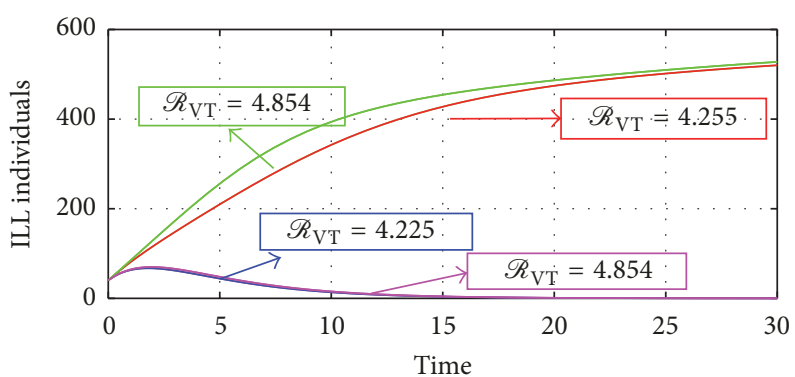

Without controls $\eta=0.65$ With controls $\eta=0.35$

With controls $\eta=0.65$

W Without controls $\eta=0.35$

(d) Optimal simulation effect in varying $\eta$ on $I(t)$

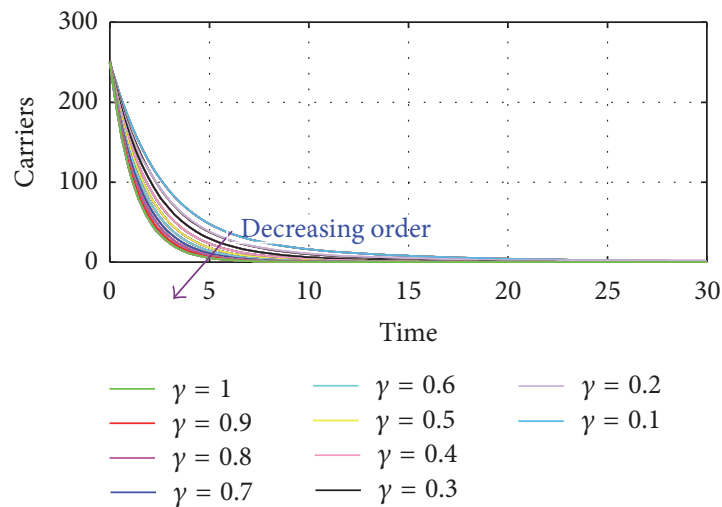

(f) Effectiveness of treatment $\gamma$ on $C(t)$

FIGURE 8: Simulation results of the optimal control model with $\eta=0.35-0.65$. The solutions for the carriers and ill individuals with $\alpha=0.2$ and $\alpha=0.5$ give $\mathscr{R}_{\mathrm{VT}}=4.225$ and $\mathscr{R}_{\mathrm{VT}}=5.250$, respectively. The case where $\eta=0.35$ and $\eta=0.65$ gives $\mathscr{R}_{\mathrm{VT}}=4.225$ and $\mathscr{R}_{\mathrm{VT}}=4.854$, respectively, which indicates the existence of an unstable disease-free equilibrium and a stable endemic equilibrium. In (e) and (f), vaccination and treatment rates are set to 0.5 and the effectiveness of the treatment control $\gamma$ varied. (e) and (f) show that as the effectiveness of the treatment control increases, the number of ill individuals reduces faster as compared to the number of carriers.

0.2 while assessing the effect of varying the rate of falling ill on carriers and on ill individuals, respectively. Hence, considering an increased rate from 0.2 to 0.7 of the rate of falling ill as shown in Figures 8(a) and 8(b) demonstrates how serious the infection is in the absence of vaccination or treatment, which indicates that ill individuals have a lower means of recovering from the disease, not even through a higher rate of natural defences of $\omega=0.5$ as indicated in Figure 8(b) without vaccination and treatment, which clearly shows that vaccination and treatment are essential in controlling meningitis irrespective of the age group; therefore we suggest that health authorities should increase the rate at which susceptible individuals get vaccination through public education and also encourage individuals with and 


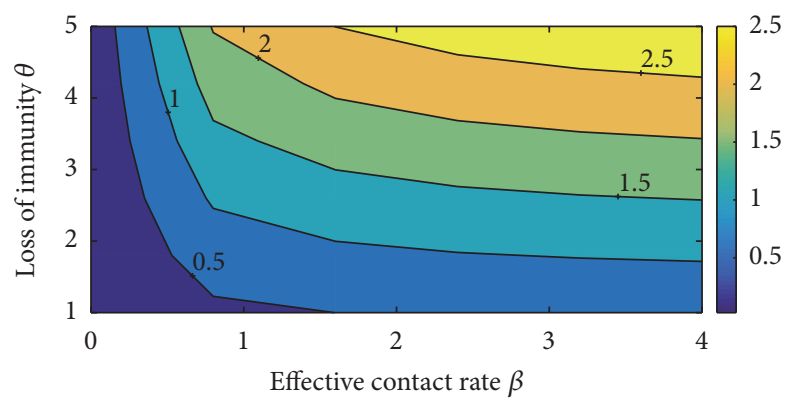

(a) The contour plot of $\theta$ and $\beta$ to $\mathscr{R}_{\mathrm{VT}}$

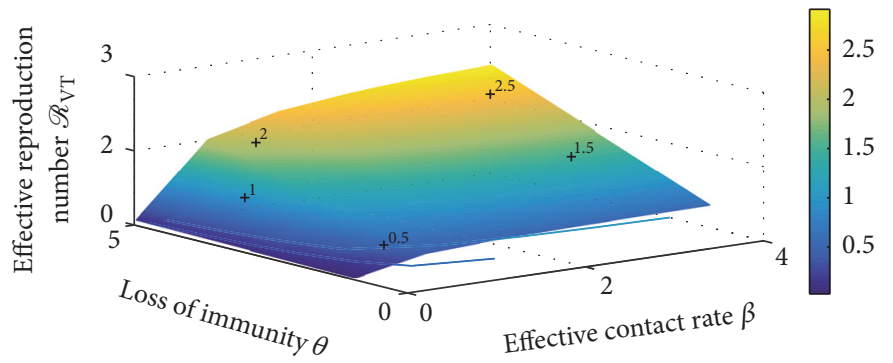

(b) The $3 \mathrm{D}$ plot of $\mathscr{R}_{\mathrm{VT}}$ to $\theta$ and $\beta$

FIGURE 9: The interepidemic period of the SCIRS model depending on parameters $\theta$ and $\beta$.

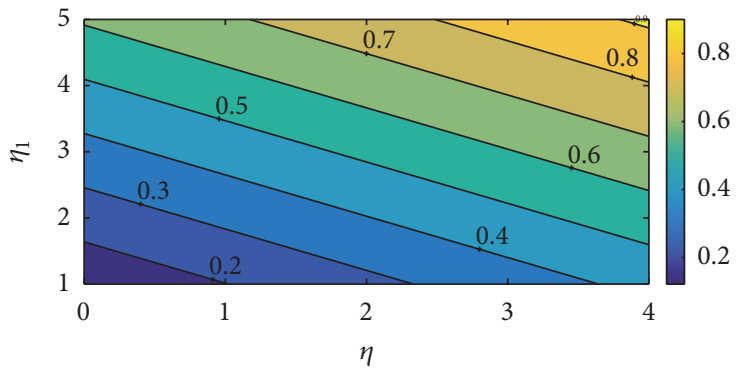

(a) The contour plot of $\eta_{1}$ and $\eta$ to $\mathscr{R}_{\mathrm{VT}}$

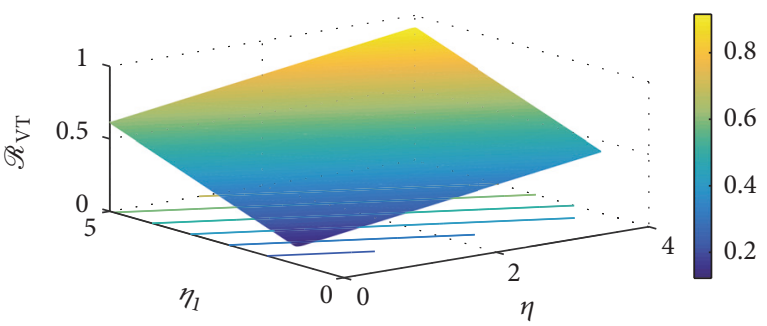

(b) The $3 \mathrm{D}$ plot of $\mathscr{R}_{\mathrm{VT}}$ to $\eta_{1}$ and $\eta$

FIGURE 10: The interepidemic period of the SCIRS model depending on parameters $\eta$ and $\eta_{1}$.

without symptoms of the infection to visit health centers for a quick check-up and if the infection is detected an immediate treatment/vaccination should be given on time to avert the spreading of the infection to community members, relatives, or close friends.

4.1. Vaccination Control Only. Figures 4(a)-4(f) show simulation results of implementing only control strategy $u_{1}$ with $u_{2}$ set to zero.

4.2. Treatment Control Only. Considering treatment control, $u_{2}$, by setting the vaccination control, $u_{1}$, to zero, the simulation results are as shown in Figures 5(a)-5(f).

4.3. Applying Both Control Measures. Finally, both control measures are implemented using the following initial population size of $S(0)=700000, C(0)=250000, I(0)=40000$, and $R(0)=10000$ and the results are plotted in Figures 6(a)-6(d).

Figures 9(a), 9(b), 10(a), and 10(b) show the interepidemic nature of some selected parameters in the SCIRS model presented above. Figures 9(a) and 9(b) indicate that as individuals lose immunity and become susceptible again, there is a corresponding increase in the rate of infection, and this suggests that an individual who receives vaccination and/or treatment and remains immune for 3-5 years should immediately go for another vaccination against the infection so as to avert transmission of the infection to that individual. Figures 10(a) and 10(b) depict that the per capita infection rate by ill individuals, $\eta_{1}$, and the per capita infection rate by carriers, $\eta$, are interlinked and so have a corresponding influence in the spread of the infection. Figure 10(b) also indicates that as the infectivity rate of carriers and ill individuals goes beyond 0.6 , there could be a high spread of the infection in the community/society should there be an outbreak, since the effective reproduction number will be getting closer to one (1) with a possibility of going beyond a unit.

\section{Conclusion}

We presented a mathematical framework of vaccination and treatment on SCIRS bacterial meningitis model. The investigation of the stability of the model shows that the diseasefree equilibrium is locally and globally asymptotically stable and the endemic equilibrium depicts a global stability. Scatter plots and the Tornado plots of the twelve parameters in $\mathscr{R}_{\mathrm{VT}}$ show that the effective contact rate $\beta$ has a major impact in transmitting the disease, followed by the infectivity potential of carriers $\eta$. This supports the fact that asymptomatic carriers are likely to have more contacts in a community/nation when there is an outbreak of bacterial meningitis compared to ill individuals who will typically be bound to their beds during the acute phase of the disease, thereby lowering the probability of infecting susceptible individuals. Finally, the numerical simulation shows that the optimal (best) way of controlling the transmission of meningitis in Sub-Saharan Africa and the world at large is to encourage susceptible 
individuals to go for vaccination against meningitis at the health centers and also report any suspected symptoms of meningitis to health practitioners for early detection and immediate care (treatment).

\section{Conflicts of Interest}

The authors declare that they have no conflicts of interest.

\section{References}

[1] W. P. Howlett, Neurology in Africa: Clinical Skills and Neurological Disorders, Cambridge University Press, 2015.

[2] M. J. F. Martínez, E. G. Merino, E. G. Sánchez, J. E. G. Sánchez, A. M. Del Rey, and G. R. Sánchez, "A mathematical model to study the meningococcal meningitis," in Proceedings of the 13th Annual International Conference on Computational Science, ICCS 2013, pp. 2492-2495, Spain, June 2013.

[3] CDC Centers for Disease Control, Bacterial Meningitis, 2017, http://www.who.int/gho/epidemic_diseases/meningitis/suspected_ cases_deaths_text/en/.

[4] K. B. Blyuss, "Mathematical modelling of the dynamics of meningococcal meningitis in Africa," UK Success Stories in Industrial Mathematics, pp. 221-226, 2016.

[5] M. C. Thigpen, C. G. Whitney, N. E. Messonnier et al., "Bacterial meningitis in the United States, 1998-2007," The New England Journal of Medicine, vol. 364, no. 21, pp. 2016-2025, 2011.

[6] CDC Centers for Disease Control, Non-polio enterovirus Infections, 2010, http://www.cdc.gov/ncidod/dvrd/revb/.

[7] B. Shmaefsky and H. Babcock, MMeningitis, Infobase Publishing, 2010.

[8] Worldbank, Meningitis vaccine provides hope to people in Ghana, 2012, http://www.who.int/features/2012/meningitis_ghana/en/.

[9] CDC Centers for Disease Control, Control and Prevention of Meningococcal Disease: Recommendations of the Advisory Committee on Immunization Practices (ACIP), 2017, https:// www.cdc.gov/mmwr/preview/mmwrhtml/00046263.htm.

[10] C. L. Trotter and M. E. Ramsay, "Vaccination against meningococcal disease in Europe: review and recommendations for the use of conjugate vaccines," FEMS Microbiology Reviews, vol. 31, no. 1, pp. 101-107, 2007.

[11] H. Broutin, S. Philippon, G. Constantin de Magny, M.-F. Courel, B. Sultan, and J.-F. Guégan, "Comparative study of meningitis dynamics across nine African countries: A global perspective," International Journal of Health Geographics, vol. 6, article no. 29, 2007.

[12] M. A. Miller and C. K. Shahab, "Review of the cost effectiveness of immunisation strategies for the control of epidemic meningococcal meningitis," PharmacoEconomics, vol. 23, no. 4, pp. 333-343, 2005.

[13] National Collaborating Centre for Women's and Children's Health, Antibiotics for Early-Onset Neonatal Infection: Antibiotics for The Prevention And Treatment of Early-Onset Neonatal Infection, RCOG Press, 2012.

[14] T. J. Irving, K. B. Blyuss, C. Colijn, and C. L. Trotter, "Modelling meningococcal meningitis in the African meningitis belt," Epidemiology and Infection, vol. 140, no. 5, pp. 897-905, 2012.

[15] B. Greenwood, "Priorities for research on meningococcal disease and the impact of serogroup A vaccination in the African meningitis belt," Vaccine, vol. 31, no. 11, pp. 1453-1457, 2013.
[16] A. C. Cohn and L. H. Harrison, "Meningococcal vaccines: Current issues and future strategies," Drugs, vol. 73, no. 11, pp. 1147-1155, 2013.

[17] R. Yogev and T. Tan, "Meningococcal disease: the advances and challenges of meningococcal disease prevention.," Human Vaccines \& Immunotherapeutics, vol. 7, no. 8, pp. 828-837, 2011.

[18] S. B. Gordon, S. Kanyanda, A. L. Walsh et al., "Poor potential coverage for 7-valent pneumococcal conjugate vaccine, Malawi," Emerging Infectious Diseases, vol. 9, no. 6, pp. 747-749, 2003.

[19] A. J. Pollard, J. Ochnio, M. Ho, M. Callaghan, M. Bigham, and S. Dobson, "Disease Susceptibility to ST11 complex meningococci bearing serogroup C or W135 polysaccharide capsules, North America," Emerging Infectious Diseases, vol. 10, no. 10, pp. 18121815, 2004.

[20] W. P. Hanage, "Serotype-specific problems associated with pneumococcal conjugate vaccination," Future Microbiology, vol. 3, no. 1, pp. 23-30, 2008.

[21] G. B. Coulson, A. Von Gottberg, M. Du Plessis, A. M. Smith, L. De Gouveia, and K. P. Klugman, "Meningococcal disease in South Africa, 1999-2002," Emerging Infectious Diseases, vol. 13, no. 2, pp. 273-281, 2007.

[22] MC. Thomson, E. Firth, M. Jancloes et al., "A climate and health partnership to inform the prevention and control of meningoccocal meningitis in sub-Saharan Africa," in the MERIT initiative. in. Climate Science for Serving Society, pp. 459-484, Springer, Climate Science for Serving Society, 2013.

[23] J. E. Mueller, L. Sangaré, B.-M. Njanpop-Lafourcade et al., "Molecular characteristics and epidemiology of meningococcal carriage, Burkina Faso, 2003," Emerging Infectious Diseases, vol. 13, no. 6, pp. 847-854, 2007.

[24] V. Dukic, M. Hayden, A. A. Forgor, and et al., "The role of weather in meningitis outbreaks in Navrongo, Ghana: a generalized additive modeling approach," Journal of Agricultural, Biological, and Environmental Statistics, vol. 17, no. 3, pp. 442460, 2012.

[25] A. Beresniak, E. Bertherat, W. Perea et al., "A Bayesian network approach to the study of historical epidemiological databases: Modelling meningitis outbreaks in the Niger," Bulletin of the World Health Organization, vol. 90, no. 6, pp. 412-416, 2012.

[26] S. Tartof, A. Cohn, F. Tarbangdo et al., "Identifying Optimal Vaccination Strategies for Serogroup A Neisseria meningitidis Conjugate Vaccine in the African Meningitis Belt," PLoS ONE, vol. 8, no. 5, Article ID e63605, 2013.

[27] L. Agier, H. Broutin, E. Bertherat et al., "Timely detection of bacterial meningitis epidemics at district level: A study in three countries of the African Meningitis Belt," Transactions of the Royal Society of Tropical Medicine and Hygiene, vol. 107, no. 1, Article ID trs010, pp. 30-36, 2013.

[28] K. Vereen, An SCIR Model of Meningococcal Meningitis, 2008.

[29] H. W. Hethcote, "Three basic epidemiological models," in Applied Mathematical Ecology, S. A. Levin, T. G. Hallam, and L. J. Gross, Eds., vol. 18 of Biomathematics, pp. 119-144, Springer, Berlin, Germany, 1989.

[30] R. L. Miller Neilan, Optimal Control Applied to Population and Disease Models (Doctoral Dissertations), 2009.

[31] A. O. Isere, J. E. Osemwenkhae, and D. Okuonghae, "Optimal control model for the outbreak of cholera in Nigeria," African Journal of Mathematics and Computer Science Research, vol. 7, no. 2, pp. 24-30, 2014. 
[32] A. A. Lashari, Mathematical Modeling And Optimal Control Of A Vector Borne Disease, National University of Modern Languages, Islamabad, Pakistan, 2012.

[33] A. Abdelrazec, S. Lenhart, and H. Zhu, "Transmission dynamics of West Nile virus in mosquitoes and corvids and non-corvids," Journal of Mathematical Biology, vol. 68, no. 6, pp. 1553-1582, 2014.

[34] A. Stashko, The effects of prevention and treatment interventions in a microeconomic model of HIV transmission, Duke University Durham, 2012.

[35] B. Seidu and O. D. Makinde, "Optimal control of HIV/AIDS in the workplace in the presence of careless individuals," Computational and Mathematical Methods in Medicine, vol. 2014, Article ID 831506, 2014.

[36] S. D. Djiomba Njankou and F. Nyabadza, "An optimal control model for Ebola virus disease," Journal of Biological Systems, vol. 24, no. 1, pp. 29-49, 2016.

[37] T. T. Yusuf and F. Benyah, "Optimal control of vaccination and treatment for an SIR epidemiological model," World Journal of Modelling and Simulation, vol. 8, no. 3, pp. 194-204, 2012.

[38] H. Gaff and E. Schaefer, "Optimal control applied to vaccination and treatment strategies for various epidemiological models," Mathematical Biosciences and Engineering, vol. 6, no. 3, pp. 469492, 2009.

[39] S. Nanda, H. Moore, and S. Lenhart, "Optimal control of treatment in a mathematical model of chronic myelogenous leukemia," Mathematical Biosciences, vol. 210, no. 1, pp. 143-156, 2007.

[40] G. Zaman, Y. Han Kang, and I. H. Jung, "Stability analysis and optimal vaccination of an SIR epidemic model," BioSystems, vol. 93, no. 3, pp. 240-249, 2008.

[41] J. K. K. Asamoah, F. T. Oduro, E. Bonyah, and B. Seidu, "Modelling of Rabies Transmission Dynamics Using Optimal Control Analysis," Journal of Applied Mathematics, vol. 2017, Article ID 2451237, 2017.

[42] U. Princeton, Emergency Guidelines for the Campus Community: Meningitis FAQ General Information, 2015, http://web.princeton .edu/sites/emergency/meningitis/FAQ-General.html.

[43] C. Sun and Y.-H. Hsieh, "Global analysis of an SEIR model with varying population size and vaccination," Applied Mathematical Modelling, vol. 34, no. 10, pp. 2685-2697, 2010.

[44] S. M. Moghadas, "Modelling the effect of imperfect vaccines on disease epidemiology," Discrete and Continuous Dynamical Systems - Series B, vol. 4, no. 4, pp. 999-1012, 2004.

[45] D. Aldila, N. Rarasati, N. Nuraini, and E. Soewono, "Optimal control problem of treatment for obesity in a closed population," International Journal of Mathematics and Mathematical Sciences, vol. 2014, Article ID 273037, 2014.

[46] M. Martcheva, An Introduction to Mathematical Epidemiology, vol. 61 of Texts in Applied Mathematics, Springer, New York, NY, USA, 2015.

[47] A. Karachaliou, A. J. K. Conlan, M.-P. Preziosi, and C. L. Trotter, "Modeling long-term vaccination strategies with MenAfriVac in the African Meningitis Belt," Clinical Infectious Diseases, vol. 61, pp. S594-S600, 2015.

[48] WHO World Health Organization, Global Health Observatory (GHO) data: Number of suspected meningitis cases and deaths reported, 2010, http://www.who.int/emergencies/diseases/en/.

[49] S. D. Hove-Musekwa, F. Nyabadza, and H. MambiliMamboundou, "Modelling hospitalization, home-based care, and individual withdrawal for people living with HIV/AIDS in high prevalence settings," Bulletin of Mathematical Biology, vol. 73, no. 12, pp. 2888-2915, 2011.

[50] C. V. De Le and C. V. De León, "Constructions of Lyapunov functions for classics SIS, SIR and SIRS epidemic model with variable population size," Foro-Red-Mat: Revista electrónica de contenido matemático, vol. 26, no. 5, p. 1, 2009.

[51] J. LaSalle, “The stability of dynamical systems," in Proceedings of the CBMS-NSF regional conference series in applied mathematics 25, Philadelphia, Pennsylvania, 1976.

[52] S. Marino, I. B. Hogue, C. J. Ray, and D. E. Kirschner, "A methodology for performing global uncertainty and sensitivity analysis in systems biology," Journal of Theoretical Biology, vol. 254, no. 1, pp. 178-196, 2008.

[53] G. Birkhoff and G.-C. Rota, Ordinary Differential Equations, Ginn, 1982.

[54] D. Iacoviello and N. Stasio, "Optimal control for SIRC epidemic outbreak," Computer Methods and Programs in Biomedicine, vol. 110, no. 3, pp. 333-342, 2013.

[55] H. Laarabi, M. Rachik, O. El Kahlaoui, and E. H. Labriji, “Optimal vaccination strategies of an SIR epidemic model with a saturated treatment," Universal Journal of Applied Mathematics, vol. 1, no. 3, pp. 185-191, 2013.

[56] S. C. Mpeshe, L. S. Luboobi, and Y. Nkansah-Gyekye, "Modeling the impact of climate change on the dynamics of rift valley fever," Computational and Mathematical Methods in Medicine, Article ID 627586, Art. ID 627586, 12 pages, 2014.

[57] D. L. Lukes, Differential Equations: Classical to Controlled, Mathematics in Science and Engineering, Academic Press, New York, NY. USA, 1982.

[58] A. A. Lashari, K. Hattaf, G. Zaman, and X. Li, "Backward bifurcation and optimal control of a vector borne disease," Applied Mathematics \& Information Sciences, vol. 7, no. 1, pp. 301-309, 2013. 


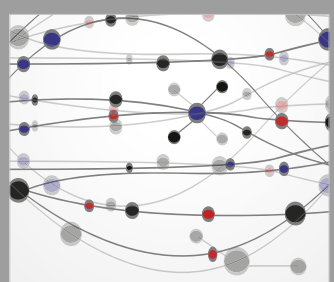

The Scientific World Journal
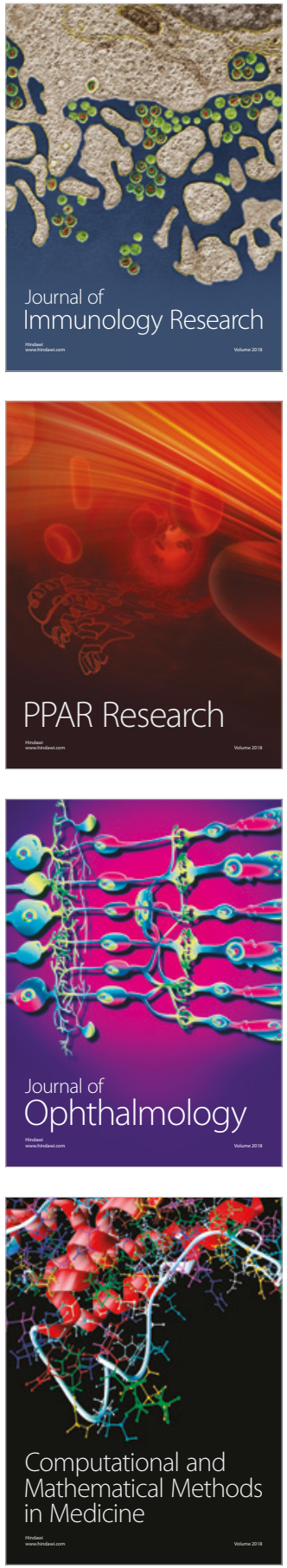

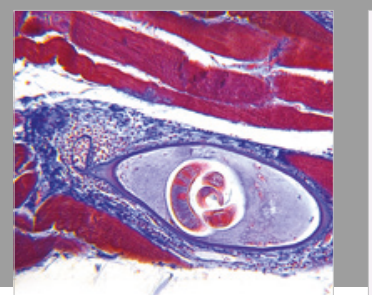

Gastroenterology Research and Practice

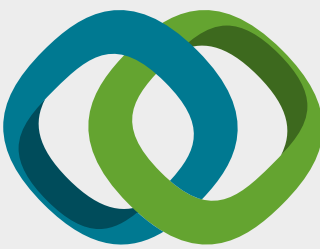

\section{Hindawi}

Submit your manuscripts at

www.hindawi.com
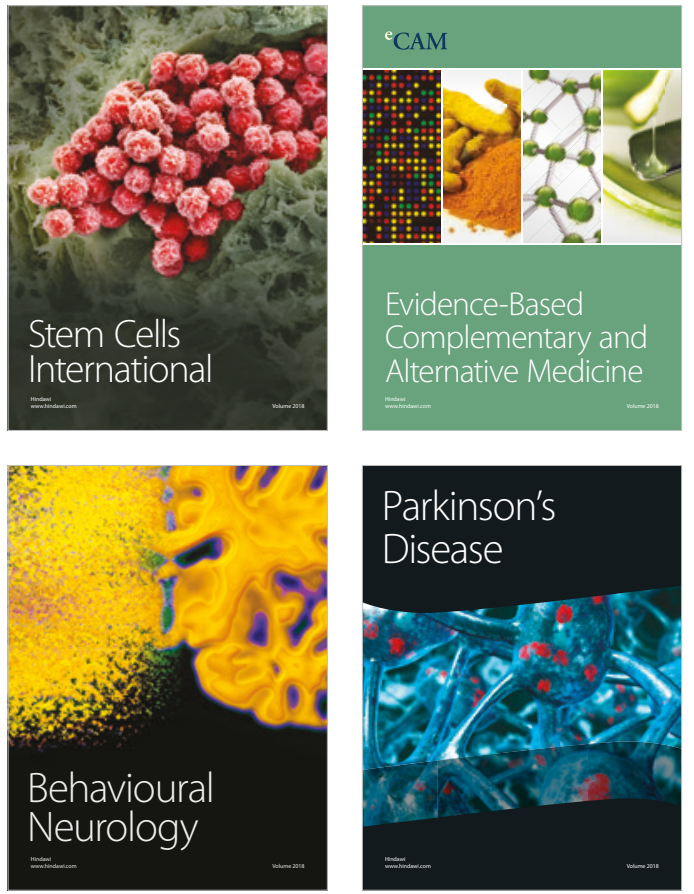

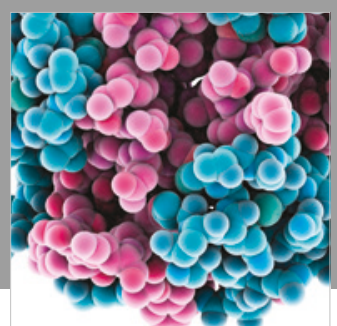

ournal of

Diabetes Research

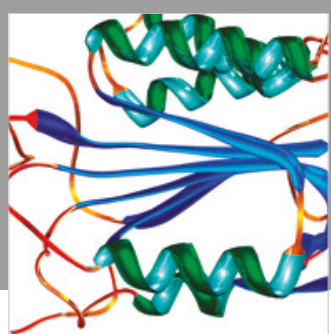

Disease Markers
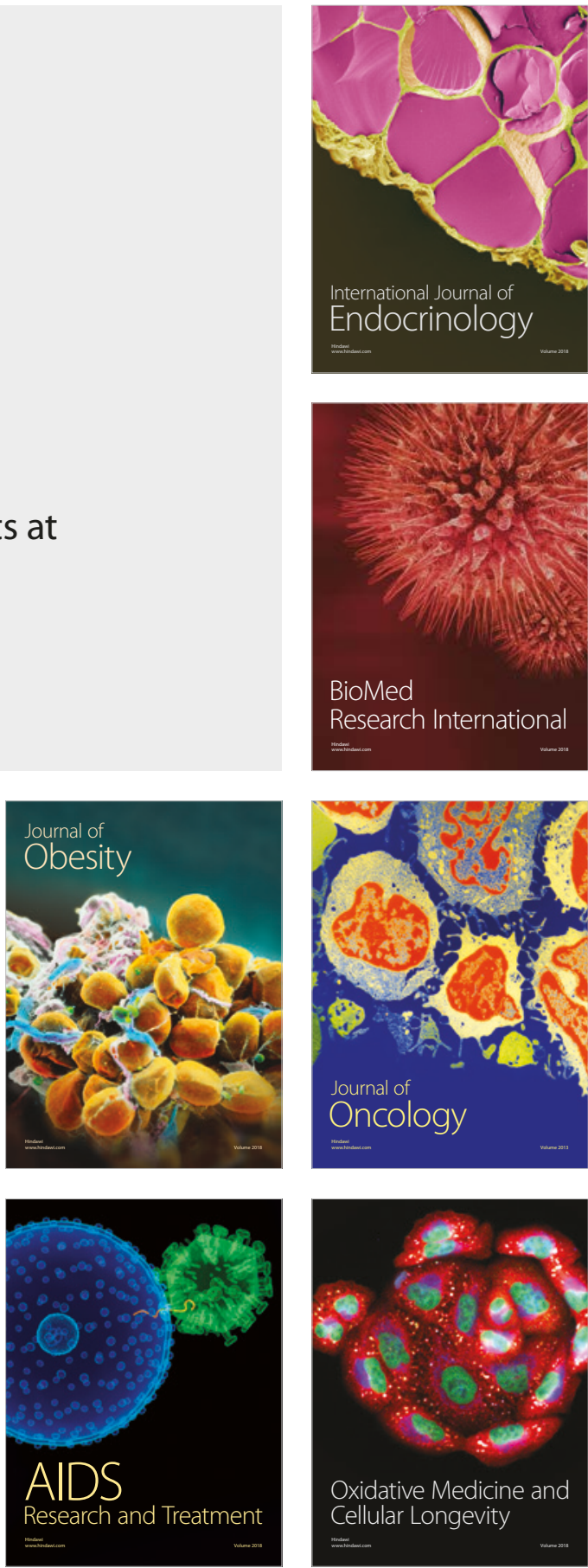